\title{
A Reverse Minkowski Theorem
}

\author{
Oded Regev ${ }^{*}$ \\ Courant Institute, New York University \\ New York, New York 10012, United States
}

\section{ABSTRACT}

We prove a conjecture due to Dadush, showing that if $\mathcal{L} \subset \mathbb{R}^{n}$ is a lattice such that $\operatorname{det}\left(\mathcal{L}^{\prime}\right) \geq 1$ for all sublattices $\mathcal{L}^{\prime} \subseteq \mathcal{L}$, then

$$
\sum_{\boldsymbol{y} \in \mathcal{L}} e^{-t^{2}\|\boldsymbol{y}\|^{2}} \leq 3 / 2,
$$

where $t:=10(\log n+2)$. From this we also derive bounds on the number of short lattice vectors and on the covering radius.

\section{CCS CONCEPTS}

- Mathematics of computing $\rightarrow$ Discrete mathematics;

\section{KEYWORDS}

Lattices, Geometry of numbers

ACM Reference format:

Oded Regev and Noah Stephens-Davidowitz. 2017. A Reverse Minkowski Theorem. In Proceedings of 49th Annual ACM SIGACT Symposium on the Theory of Computing, Montreal, Canada, June 2017 (STOC'17), 13 pages.

DOI: $10.1145 / 3055399.3055434$

\section{INTRODUCTION}

A lattice $\mathcal{L} \subset \mathbb{R}^{n}$ is the set of integer linear combinations of linearly independent basis vectors $\mathbf{B}=\left(\boldsymbol{b}_{1}, \ldots, \boldsymbol{b}_{n}\right)$. The determinant of the lattice, $\operatorname{det}(\mathcal{L})=|\operatorname{det}(\mathbf{B})|$, is a measure of its global density in the sense that

$$
\operatorname{det}(\mathcal{L})=\lim _{r \rightarrow \infty} \frac{\operatorname{vol}\left(r B_{2}^{n}\right)}{\left|\mathcal{L} \cap r B_{2}^{n}\right|},
$$

where $r B_{2}^{n}$ denotes the closed Euclidean ball of radius $r>0$, whose volume is $(\pi n)^{-1 / 2}\left(2 \pi e r^{2} / n\right)^{n / 2}(1+o(1))$. (Here and elsewhere, we write $o(1)$ for an arbitrary function that approaches zero as the dimension $n$ approaches infinity.)

\footnotetext{
*Supported by the Simons Collaboration on Algorithms and Geometry and by the National Science Foundation (NSF) under Grant No. CCF-1320188. Any opinions, findings, and conclusions or recommendations expressed in this material are those of the authors and do not necessarily reflect the views of the NSF.

${ }^{\dagger}$ Supported by the National Science Foundation (NSF) under Grant No. CCF-1320188, and the Defense Advanced Research Projects Agency (DARPA) and Army Research Office (ARO) under Contract No. W911NF-15-C-0236. Part of this work was done while visiting Chris Peikert at the University of Michigan.
}

Permission to make digital or hard copies of all or part of this work for personal or classroom use is granted without fee provided that copies are not made or distributed for profit or commercial advantage and that copies bear this notice and the full citation on the first page. Copyrights for components of this work owned by others than ACM must be honored. Abstracting with credit is permitted. To copy otherwise, or republish, to post on servers or to redistribute to lists, requires prior specific permission and/or a fee. Request permissions from permissions@acm.org.

STOC'17, Montreal, Canada

(c) 2017 ACM. 978-1-4503-4528-6/17/06 ..\$15.00

DOI: $10.1145 / 3055399.3055434$

\author{
Noah Stephens-Davidowitz ${ }^{\dagger}$ \\ Courant Institute, New York University \\ New York, New York 10012, United States \\ noahsd@gmail.com
}

Minkowski's celebrated first theorem shows that a lattice with small determinant must have short non-zero vectors [31]. This is one of the foundational results in the study of lattices and the geometry of numbers, and it has innumerable applications. We consider the following point-counting form of this theorem due to Blichfeldt and van der Corput, ${ }^{1}$ which says that a lattice with small determinant must have many short points, or informally, that "global density implies local density."

Theorem 1.1 ([40]). For any lattice $\mathcal{L} \subset \mathbb{R}^{n}$ with $\operatorname{det}(\mathcal{L}) \leq 1$ and $r>0$,

$$
\left|\mathcal{L} \cap r B_{2}^{n}\right| \geq 2^{-n} \cdot \operatorname{vol}\left(r B_{2}^{n}\right)=\frac{1}{\sqrt{\pi n}}\left(\frac{\pi e r^{2}}{2 n}\right)^{n / 2}(1+o(1)) .
$$

It is quite natural to ask whether a converse of Theorem 1.1 holds. In particular, if a lattice has sufficiently many short points, does it necessarily have small determinant? Does local density imply global density?

It is easy to see that the answer is actually no. Consider, for example, the lattice generated by the vectors $(1 / t, 0)$ and $\left(0, t^{2}\right)$ for some arbitrarily large $t$. This lattice has at least $2\lfloor t r\rfloor+1$ points of norm at most $r$, but it has arbitrarily large determinant $t$. Notice, however, that this lattice contains a sublattice generated by $(1 / t, 0)$ that does have small determinant. This leads us to a more refined question:

If a lattice has sufficiently many short points, does it necessarily have a small-determinant sublattice? Does local density imply global density over a subspace?

Equivalently, in the contrapositive, the question asks for an upper bound on the number of lattice points in a ball given that there is no sublattice of small determinant.

Dadush conjectured a suitably precise answer to these questions [11]. He later studied this conjecture in depth in joint work with the first named author [13]. Among other things, they showed a number of applications of the conjecture (from computational complexity of lattice problems to Brownian motion on flat tori) and gave some evidence for it. We refer the reader to [13] for a full list of their results.

Our main result is a proof of the conjecture of Dadush, which in particular implies the applications mentioned above.

Theorem 1.2 (Reverse Minkowski Theorem). For any lattice $\mathcal{L} \subset \mathbb{R}^{n}$ with $\operatorname{det}\left(\mathcal{L}^{\prime}\right) \geq 1$ for all sublattices $\mathcal{L}^{\prime} \subseteq \mathcal{L}$,

$$
\rho_{1 / t}(\mathcal{L}) \leq \frac{3}{2}
$$

where $t:=10(\log n+2)$.

${ }^{1}$ They actually showed the slightly stronger bound $\left|\mathcal{L} \cap r B_{2}^{n}\right| \geq 2\left\lfloor 2^{-n} \cdot \operatorname{vol}\left(r B_{2}^{n}\right)\right\rfloor+1$ and considered arbitrary norms, not just $\ell_{2}$. (See, e.g., [18, Thm. 1 of Ch. 2, Sec. 7].) 
Here, for a lattice $\mathcal{L} \subset \mathbb{R}^{n}$ and $s>0$,

$$
\rho_{s}(\mathcal{L}):=\sum_{\boldsymbol{y} \in \mathcal{L}} e^{-\pi\|\boldsymbol{y}\|^{2} / s^{2}}
$$

is the Gaussian mass of the lattice with parameter $s$. This can be seen as a smooth version of the point-counting function $r \mapsto \mid \mathcal{L} \cap$ $r B_{2}^{n} \mid$, with the parameter $s$ playing the role of the radius $r$, and it arises naturally in a number of contexts (often in the form of the theta function, $\Theta_{\mathcal{L}}(i y):=\rho_{1 / \sqrt{y}}(\mathcal{L})$ ). In particular, Theorem 1.2 immediately implies that $\left|\mathcal{L} \cap r B_{2}^{n}\right| \leq 3 e^{\pi t^{2} r^{2}} / 2$ for any radius $r>0$.

We can equivalently formulate Theorem 1.2 in terms of the parameter

$$
\eta^{*}(\mathcal{L}):=\inf \left\{t: \rho_{1 / t}(\mathcal{L}) \leq 3 / 2\right\}
$$

(known as the smoothing parameter of the dual lattice [30]). Namely, by scaling $\mathcal{L}$ so that

$$
\eta_{\text {det }}(\mathcal{L}):=\max _{\mathcal{L}^{\prime} \subseteq \mathcal{L}} \operatorname{det}\left(\mathcal{L}^{\prime}\right)^{-1 / \operatorname{rank}\left(\mathcal{L}^{\prime}\right)}
$$

is equal to one, Theorem 1.2 shows that

$$
\frac{2}{3} \cdot \eta_{\operatorname{det}}(\mathcal{L}) \leq \eta^{*}(\mathcal{L}) \leq 10(\log n+2) \cdot \eta_{\operatorname{det}}(\mathcal{L}),
$$

where the lower bound is an immediate consequence of the Poisson Summation Formula (Eq. (3)). For example, $\eta^{*}\left(\mathbb{Z}^{n}\right)=\sqrt{\log n / \pi}+$ $o(1)$ and $\eta_{\text {det }}\left(\mathbb{Z}^{n}\right)=1$.

In the full version of this work [34], we extend Theorem 1.2 to obtain a bound on the Gaussian mass for all parameters, as follows.

Theorem 1.3. For any lattice $\mathcal{L} \subset \mathbb{R}^{n}$ with $\operatorname{det}\left(\mathcal{L}^{\prime}\right) \geq 1$ for all sublattices $\mathcal{L}^{\prime} \subseteq \mathcal{L}$,

(1) $\rho_{s}(\mathcal{L}) \leq 1+e^{-\pi\left(1 / s^{2}-t^{2}\right)} / 2$ for any $s \leq 1 / t$;

(2) $\rho_{s}(\mathcal{L}) \leq(C s t)^{n / 2}$ for any $1 / t<s<t$ and some universal constant $C>1$; and

(3) $\rho_{s}(\mathcal{L}) \leq 2 s^{n}$ for any $s \geq t$,

where $t:=10(\log n+2)$.

Theorem 1.3 implies the following point-counting bounds. (See the full version [34] for the proof.)

COROllary 1.4. For every lattice $\mathcal{L} \subset \mathbb{R}^{n}$ with $\operatorname{det}\left(\mathcal{L}^{\prime}\right) \geq 1$ for all sublattices $\mathcal{L}^{\prime} \subseteq \mathcal{L}$, and every shift vector $\boldsymbol{u} \in \mathbb{R}^{n}$,

(1) for any $r \geq 1,\left|\mathcal{L} \cap\left(r B_{2}^{n}+\boldsymbol{u}\right)\right| \leq 3 e^{\pi t^{2} r^{2}} / 2$;

(2) for any $\sqrt{n /(2 \pi)} \cdot t^{-1} \leq r \leq \sqrt{n /(2 \pi)} \cdot t,\left|\mathcal{L} \cap\left(r B_{2}^{n}+\boldsymbol{u}\right)\right| \leq$ $(C t r / \sqrt{n})^{n / 2}$ for some universal constant $C>0$; and

(3) for any $r \geq \sqrt{n /(2 \pi)} \cdot t,\left|\mathcal{L} \cap\left(r B_{2}^{n}+\boldsymbol{u}\right)\right| \leq 2\left(2 \pi e r^{2} / n\right)^{n / 2}$, where $t:=10(\log n+2)$.

In the full version [34], we discuss the tightness of Theorem 1.3 and Corollary 1.4 .

\subsection{Approximation to the Covering Radius}

The covering radius $\mu(\mathcal{L})$ of a lattice $\mathcal{L} \subset \mathbb{R}^{n}$ is the maximal distance from any point in $\mathbb{R}^{n}$ to the lattice, or equivalently, the minimum radius $r$ such that $\mathcal{L}+r B_{2}^{n}=\mathbb{R}^{n}$. It follows from the definition that $\mu(\mathcal{L})$ must be at least the radius of a ball of volume $\operatorname{det}(\mathcal{L})$, which is at least $\sqrt{n /(2 \pi e)} \operatorname{det}(\mathcal{L})^{1 / n}$. By considering projections, Kannan and Lovász [24] improved this lower bound, as follows. Let $\pi_{W^{\perp}}(\mathcal{L})$ be the projection of the lattice onto the space $W^{\perp}$ orthogonal to some lattice subspace $W \subset \mathbb{R}^{n}$-a subspace spanned by $k<n$ linearly independent lattice vectors. ${ }^{2}$ Then clearly $\mu(\mathcal{L}) \geq \mu\left(\pi_{W^{\perp}}(\mathcal{L})\right)$, and the latter is at least $\left(\operatorname{dim}\left(W^{\perp}\right) /(2 \pi e)\right)^{1 / 2}$. $\operatorname{det}\left(\pi_{W^{\perp}}(\mathcal{L})\right)^{1 / \operatorname{dim}\left(W^{\perp}\right)}$. So, we obtain the lower bound

$$
\mu(\mathcal{L}) \geq \frac{1}{\sqrt{2 \pi e}} \cdot \mu_{\mathrm{det}}(\mathcal{L})
$$

where

$$
\begin{aligned}
\mu_{\operatorname{det}}(\mathcal{L}) & :=\max _{W \subset \mathbb{R}^{n}} \sqrt{\operatorname{dim}\left(W^{\perp}\right)} \cdot \operatorname{det}\left(\pi_{W^{\perp}}(\mathcal{L})\right)^{\frac{1}{\operatorname{dim}\left(W^{\perp}\right)}} \\
& =\max _{\mathcal{M} \subseteq \mathcal{L}^{*}} \sqrt{\operatorname{rank}(\mathcal{M})} \cdot \operatorname{det}(\mathcal{M})^{-\frac{1}{\operatorname{rank}(\mathcal{M})}},
\end{aligned}
$$

with the first maximum taken over lattice subspaces $W \subset \mathbb{R}^{n}$ and $\mathcal{L}^{*}$ being the dual lattice. Kannan and Lovász also observed the upper bound

$$
\mu(\mathcal{L}) \leq C \sqrt{n} \cdot \mu_{\operatorname{det}}(\mathcal{L})
$$

(see [13, Theorem 11.1] for a proof), and asked whether a better upper bound could be found. ${ }^{3}$ In Section 5, we use Theorem 1.2 to derive the following improved bound.

Theorem 1.5 (COVERING-RAdius APproximation). For any lattice $\mathcal{L} \subset \mathbb{R}^{n}$,

$$
\frac{1}{\sqrt{2 \pi e}} \cdot \mu_{\mathrm{det}}(\mathcal{L}) \leq \mu(\mathcal{L}) \leq 10(\log n+10)^{3 / 2} \cdot \mu_{\mathrm{det}}(\mathcal{L}) .
$$

We emphasize that Dadush and Regev [13] already proved that Theorem 1.5 (with slightly weaker parameters) would follow from a proof of Theorem 1.2. Although our proof is shorter and achieves slightly better parameters, it is conceptually similar to the one in [13].

We note that the specific polylogarithmic factor that we obtain is likely not optimal. In fact, in Theorem 5.8 we prove a bound similar to that in Eq. (2) that replaces the factor $10(\log n+10)^{3 / 2}$ by $C \sqrt{\log n}$, assuming the celebrated Slicing Conjecture [5, 25]. However, it is not difficult to show that this factor cannot be smaller than $\sqrt{\log n /(4 e)}+o(1){ }^{4}$

Covering Radius of Stable Lattices and Minkowski's Conjecture. We say that a lattice $\mathcal{L} \subset \mathbb{R}^{n}$ is stable if $\operatorname{det}(\mathcal{L})=1$ and $\operatorname{det}\left(\mathcal{L}^{\prime}\right) \geq 1$ for all sublattices $\mathcal{L}^{\prime} \subseteq \mathcal{L}$. Stable lattices arise in a number of contexts $[16,20,38]$ and they play an important role in the sequel. Shapira and Weiss showed that a tight bound of $\mu(\mathcal{L}) \leq \mu\left(\mathbb{Z}^{n}\right)=$ $\sqrt{n} / 2$ on the covering radius of stable lattices would imply a wellknown conjecture attributed to Minkowski [36]. (See also [37].) We do not manage to prove such a tight bound, but en route to proving Theorem 1.5 we do show that $\mu(\mathcal{L}) \leq 4 \sqrt{n}(\log n+10)$ for all stable lattices. (See Theorem 5.2.) We also observe that a very strong resolution to the Slicing Conjecture and a better bound between two lattice parameters would yield the desired tight bound. (See Theorem 5.7 and the discussion afterwards.)

\footnotetext{
${ }^{2}$ The projection $\pi_{W^{\perp}}(\mathcal{L})$ is a lattice if and only if $W$ is a lattice subspace. ${ }^{3}$ They also proved similar bounds for arbitrary norms [24, Corollary 3.11]. ${ }^{4}$ Consider the lattice $\mathcal{L}$ generated by $\left(\boldsymbol{e}_{1}, \boldsymbol{e}_{2} / 2,2 \boldsymbol{e}_{3} / 3^{3 / 2}, \ldots,(n-\right.$ 1) $\left.{ }^{(n-1) / 2} e_{n} / n^{n / 2}\right)$. It is not difficult to verify that $\mu_{\operatorname{det}}(\mathcal{L})=1$, but $\mu(\mathcal{L})=\sqrt{\log n /(4 e)}+o(1)$.
} 


\subsection{An Optimal Bound on the Gaussian Mass for "Extreme" Parameters}

It is tempting to ask whether $\rho_{s}(\mathcal{L}) \leq \rho_{s}\left(\mathbb{Z}^{n}\right)$ for any lattice $\mathcal{L} \subset$ $\mathbb{R}^{n}$ such that $\operatorname{det}\left(\mathcal{L}^{\prime}\right) \geq 1$ for all sublattices $\mathcal{L}^{\prime} \subseteq \mathcal{L}$ and any parameter $s>0$. (See Section 1.5.) The next theorem shows that indeed $\rho_{s}(\mathcal{L}) \leq \rho_{s}\left(\mathbb{Z}^{n}\right)$ for such lattices, but only for "extremely low" or "extremely high" parameters $s$. The proof is in the full version of this work [34].

Theorem 1.6. For any lattice $\mathcal{L} \subset \mathbb{R}^{n}$ such that $\operatorname{det}\left(\mathcal{L}^{\prime}\right) \geq 1$ for all sublattices $\mathcal{L}^{\prime} \subseteq \mathcal{L}$ and parameter $s>0$ such that either $s \leq \sqrt{2 \pi /(n+2)}$ or $s \geq \sqrt{(n+2) /(2 \pi)}$, we have $\rho_{s}(\mathcal{L}) \leq \rho_{s}\left(\mathbb{Z}^{n}\right)$.

We hope that the proof of Theorem 1.6 might provide some hints as to how to extend it to all parameters $s$.

\subsection{Proof Overview}

In this section, we give a high-level overview of the proof of Theorem 1.2 .

Bounding the Mass of Stable Lattices. Recall that a lattice $\mathcal{L}$ is stable if $\operatorname{det}(\mathcal{L})=1$ and $\operatorname{det}\left(\mathcal{L}^{\prime}\right) \geq 1$ for all sublattices $\mathcal{L}^{\prime} \subseteq$ $\mathcal{L}$. I.e., stable lattices are determinant-one lattices that satisfy the assumption in Theorem 1.2. In this proof overview, we focus on bounding the Gaussian mass $\rho_{s}(\mathcal{L})$ of stable lattices $\mathcal{L}$. As it turns out, the general case then follows easily.

Crucially, the stable lattices form a compact subset of the set of determinant-one lattices, so that the continuous function $\rho_{s}(\mathcal{L})$ must attain a global maximum over the set of stable lattices. We may therefore restrict our attention to a lattice that corresponds to this global maximum. If this lattice is on the boundary of the set of stable lattices, then it has a strict sublattice $\mathcal{L}^{\prime}$ with determinant one. We can then "split the lattice" at $\mathcal{L}^{\prime}$. Namely, we can replace the original lattice $\mathcal{L}$ by the direct sum $\mathcal{L}^{\prime} \oplus \mathcal{L} / \mathcal{L}^{\prime}$. It is not difficult to prove that

$$
\rho_{s}(\mathcal{L}) \leq \rho_{s}\left(\mathcal{L}^{\prime} \oplus \mathcal{L} / \mathcal{L}^{\prime}\right)=\rho_{s}\left(\mathcal{L}^{\prime}\right) \rho_{s}\left(\mathcal{L} / \mathcal{L}^{\prime}\right)
$$

and that $\mathcal{L}^{\prime}$ and $\mathcal{L} / \mathcal{L}^{\prime}$ are stable. So, we have reduced the question to a lower-dimensional one. Therefore, if we could show that for any dimension, the global maximizer is on the boundary, then we could use induction to show that the global maximizer of the Gaussian mass is simply the integer lattice $\mathbb{Z}^{n}=\mathbb{Z} \oplus \cdots \oplus \mathbb{Z}$.

Indeed, this is how we prove Theorem 1.6 (in the full version [34]), which shows that $\mathbb{Z}^{n}$ has maximal Gaussian mass for certain "extreme" parameters $s$. For such parameters, by taking the second derivative, we show that a stable lattice cannot be a local maximum over the set of determinant-one lattices. Therefore, the global maximizer of $\rho_{S}(\mathcal{L})$ over the compact subset of stable lattices must be on the boundary, and we can perform the "splitting" procedure described above to show by induction that $\rho_{s}(\mathcal{L}) \leq \rho_{s}\left(\mathbb{Z}^{n}\right)$.

However, we do not know if $\rho_{s}(\mathcal{L})$ can have such stable local maxima for other parameters. As a potential way around this issue, we could use a natural and very elegant idea due to Shapira and Weiss [36]-We could try to directly bound the value of $\rho_{S}(\mathcal{L})$ at any hypothetical local maximum. Then, either the global maximum of $\rho_{s}(\mathcal{L})$ over the set of stable lattices is one of these local maxima, in which case we can apply this bound; or it is on the boundary, in which case we can "split the lattice" as above. (Shapira and Weiss suggested using this approach to bound the covering radius of stable lattices to resolve Minkowski's Conjecture [36]. Interestingly, local maxima of the covering radius do exist [14].)

Enter the Voronoi Cell. Unfortunately, even bounding the value of $\rho_{s}(\mathcal{L})$ at local maxima seems to be beyond our grasp. So, instead of working with $\rho_{s}(\mathcal{L})$ directly, we work with a proxy for it: the Gaussian mass of the Voronoi cell of the lattice

$$
\gamma_{s}(\mathcal{V}(\mathcal{L})):=\int_{\mathcal{V}(\mathcal{L}) / s} e^{-\pi\|x\|^{2}} \mathrm{~d} \boldsymbol{x}
$$

where the Voronoi cell is the set of all points that are closer to the origin than to any other lattice vector

$$
\mathcal{V}(\mathcal{L}):=\left\{\boldsymbol{x} \in \mathbb{R}^{n}: \forall \boldsymbol{y} \in \mathcal{L},\|\boldsymbol{x}\| \leq\|\boldsymbol{y}-\boldsymbol{x}\|\right\} .
$$

An elegant proof due to Chung, Dadush, Liu, and Peikert [7] shows that $\rho_{s}(\mathcal{L})$ is at most $1 / \gamma_{s}(\mathcal{V}(\mathcal{L}))$. (See Lemma 4.1.) So, in order to prove an upper bound on $\rho_{S}(\mathcal{L})$, it suffices to prove a lower bound on $\gamma_{s}(\mathcal{V}(\mathcal{L}))$.

We accomplish this via the approach described above. I.e., we reduce the problem to bounding the value of $\gamma_{s}(\mathcal{V}(\mathcal{L}))$ at local minima. (Here too, we do not know whether these local minima exist.) By comparing gradients, we then show (in Section 3) that any lattice corresponding to a local minimum must have a Voronoi cell $\mathcal{V}(\mathcal{L})$ such that the function $A \mapsto \gamma_{S}(A \mathcal{V}(\mathcal{L}))$ has a critical point at $A=I_{n}$, where $A \in \mathrm{SL}_{n}(\mathbb{R})$ ranges over all determinant-one matrices. Using a result due to Bobkov [3], which itself follows from a deep theorem due to Cordero-Erausquin, Fradelizi, and Maurey $[10],{ }^{5}$ we can show that any such critical point must actually be a global maximum of the function $A \mapsto \gamma_{S}(A \mathcal{V}(\mathcal{L}))$. I.e., in the language of convex geometry, the Voronoi cell is in a position that maximizes the Gaussian mass. (Note the rather surprising jump from a presumed local minimum over the set of determinant-one lattices to a global maximum over the set of positions of the Voronoi cell.)

Finally, we complete the proof by applying the celebrated $\ell \ell^{*}$ theorem $[15,28,33]$. In particular, this theorem tells us that every convex body $K$ with $\operatorname{vol}(K)=1$ has a position $A \in \mathrm{SL}_{n}(\mathbb{R})$ such that $\gamma_{1 / t}(A K) \geq 2 / 3$, with $t:=10(\log n+2)$ as in Theorem 1.2. (See Theorem 4.6.) Since the Voronoi cell is already in a position that maximizes the mass, we must have $\gamma_{1 / t}(\mathcal{V}(\mathcal{L})) \geq \gamma_{1 / t}(A \mathcal{V}(\mathcal{L})) \geq$ $2 / 3$. We then obtain the desired bound on $\rho_{1 / t}(\mathcal{L})$ by applying the result of [7].

\subsection{Related Work}

Our main theorem was originally conjectured by Dadush [11]. Dadush together with the first named author described several applications of the conjecture [13]. In particular, they showed the connection between this conjecture and the Kannan-Lovász-style covering-radius approximation given in Theorem 1.5. They also used a result from convex geometry (specifically the Milman-Pisier Theorem) as evidence for the conjecture. That theorem is related to the $\ell \ell^{*}$ theorem that we use in our proof.

The high-level outline of our proof (in which we obtain a bound on a lattice parameter by reducing the question to stable local

\footnotetext{
${ }^{5}$ We note in passing that one can prove Theorem 1.2 (at least up to constants) without using this rather heavy hammer by considering local maxima of the $\ell$-norm of the Voronoi cell instead of local minima of the Gaussian mass of the Voronoi cell.
} 
extrema) is due to Shapira and Weiss [36]. They showed that an important conjecture attributed to Minkowski would follow if we could prove that $\mathbb{Z}^{n}$ has maximal covering radius amongst all stable lattices (i.e., that the covering radius of an $n$-dimensional stable lattice is at most $\sqrt{n} / 2$ ). They then observed that it would suffice to bound the covering radius of the lattices corresponding to local maxima of the covering radius function over the set of determinantone lattices.

Stable lattices were introduced (in a more general context) by Harder and Narasimhan [20] and by Stuhler [38]. Our presentation more-or-less follows that of Grayson [16].

Counting the number of lattice points in a ball is a classical question, and a summary of all that is known is far beyond the scope of this paper. (See, e.g., [9].) In particular, much research has gone into studying the relationship between the number of points in a ball of radius $r$ and the determinant of the densest onedimensional sublattice, written $\lambda_{1}(\mathcal{L})$. (I.e., $\lambda_{1}(\mathcal{L})$ is the length of the shortest non-zero vector in the lattice.) It is easy to see that the number of lattice points in a ball of radius $r \geq \lambda_{1}(\mathcal{L})$ is at most $\left(C r / \lambda_{1}(\mathcal{L})\right)^{n}$, which is essentially the best possible bound based on $\lambda_{1}(\mathcal{L}) .{ }^{6}$ We consider the densest sublattice of any dimension (not just the densest one-dimensional sublattice) to obtain bounds that are much stronger in many cases. (Other authors have considered other generalizations of $\lambda_{1}(\mathcal{L})$ to derive incomparable bounds. E.g., [22].)

Many authors have considered the extrema of various lattice parameters over the set of determinant-one lattices. Voronoi famously characterized the local maxima of the length of the shortest non-zero vector [41], and a long line of work has gone into finding the specific global maxima in various dimensions. (See, e.g., $[8,9]$.) Similarly, Montgomery [32] and Sarnak and Strömbergsson [35] considered the minima of the Gaussian mass $\rho_{s}(\mathcal{L})$ and closely related functions.

Informally, the results mentioned above (and almost all literature on this topic since Minkowski) were concerned with the "best" lattices. E.g., the largest minimum distance, the smallest covering radius, the minimal Gaussian mass, etc. We are in some sense interested in the "worst" lattices. Thus, we consider maxima of the Gaussian mass, maxima of the covering radius (as in [14]), etc. (These questions only makes sense over a bounded subset of the determinant-one lattices, such as the stable lattices.) Note that, while the "best" lattices tend to have fascinating properties (see, e.g., [9]), in our setting the "worst" lattice that we know of is $\mathbb{Z}^{n}$.

In recent follow-up work, Lovett and the first named author used Theorem 1.2 to give a counterexample to a very strong variant of the polynomial Frieman-Ruzsa conjecture over the integers [29]. This variant was introduced by Green (who suggested that it was likely to be false) [17].

\subsection{Directions for Future Work}

The most obvious direction for future work is to try to obtain a better value for $t$ in Theorem 1.2. As far as we know, the correct value could be as small as $t=\eta^{*}\left(\mathbb{Z}^{n}\right)=\sqrt{\log (n) / \pi}+o(1)$. Our proof

\footnotetext{
${ }^{6}$ Finding the exact best possible bounds on $\left|\mathcal{L} \cap s \lambda_{1}(\mathcal{L}) B_{2}^{n}\right|$ in various regimes is a fascinating classical problem that is still an active area of research. For example, when $s=1$, this is known as the lattice "kissing number" problem, and the limit as $s \rightarrow \infty$ is the lattice sphere-packing problem. See, e.g., $[8,9,23]$.
}

seems to be loose in two places: (1) Theorem 4.6, which bounds the maximal Gaussian mass of convex bodies; and (2) the induction argument in the proof of Proposition 4.14. It seems that one would need to improve both parts of the proof to obtain a significantly better bound.

A more ambitious goal would be to prove that $\mathbb{Z}^{n}$ is the exact maximizer in Theorem 1.3 for all parameters $s>0$. One might try to prove this by showing that $\rho_{s}(\mathcal{L})$ has no local maxima over the set of determinant-one lattices for any parameter $s>0$. Alternatively, one can try using the technique of "characterizing the local extrema" that we use to prove Theorem 1.2. For this, we note that any local maximum of $\rho_{s}(\mathcal{L})$ must correspond to an "isotropic" lattice $\mathcal{L}$ in the sense that

$$
\sum_{\boldsymbol{y} \in \mathcal{L}} \rho_{s}(\boldsymbol{y}) \boldsymbol{y} \boldsymbol{y}^{T}=\alpha \cdot I_{n}
$$

for some scalar $\alpha>0$. So, it would suffice to show that $\rho_{S}(\mathcal{L}) \leq$ $\rho_{s}\left(\mathbb{Z}^{n}\right)$ for (stable) "isotropic" lattices. Unfortunately, we do not know how to make use of this.

Recall from Eq. (1) that Theorem 1.2 gives quite a tight approximation to the smoothing parameter $\eta^{*}(\mathcal{L})$. However, an analogous tightness result does not hold for Theorem 1.3 and Corollary 1.4. Dadush and Regev therefore suggested a potential refinement that depends on "the full spectrum of dense sublattices," $\min _{\mathcal{L}^{\prime} \subseteq \mathcal{L}, \operatorname{rank}\left(\mathcal{L}^{\prime}\right)=k} \operatorname{det}\left(\mathcal{L}^{\prime}\right)^{1 / k}$ for $k=1, \ldots, n$, rather than just

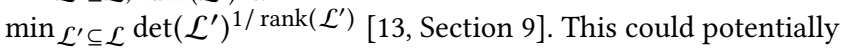
give a tight characterization of $\left|\mathcal{L} \cap r B_{2}^{n}\right|$ for all radii $r$ and all lattices $\mathcal{L} \subset \mathbb{R}^{n}$.

One can also consider generalizations of Theorems 1.5 and 1.2 to arbitrary norms, as discussed in [24] and [13, Section 9] respectively. Extending Theorem 1.5 to arbitrary norms could potentially yield faster algorithms for Integer Programming [12]. Unfortunately, a natural generalization of Theorem 1.2 actually fails. (See [13, Section 9].)

\section{PRELIMINARIES}

We use $c, C, C^{\prime}$ to denote arbitrary positive universal constants, whose value might change from one occurrence to the next. Logarithms are base $e$ unless otherwise specified. Vectors $x \in \mathbb{R}^{n}$ are column vectors. We write $\|\boldsymbol{x}\|$ to represent the Euclidean norm of $\boldsymbol{x}$, and we write $I_{n}$ for the identity matrix in $n$ dimensions. For a matrix $A \in \mathbb{R}^{n \times n}$, we write $A^{T}$ for the transpose of $A$. We write $B_{2}^{n}:=\left\{\boldsymbol{x} \in \mathbb{R}^{n}:\|\boldsymbol{x}\| \leq 1\right\}$ for the Euclidean ball in $\mathbb{R}^{n}$. We write $\pi_{S}(\boldsymbol{x})$ for the orthogonal projection of $\boldsymbol{x}$ onto $\operatorname{span}(S)$ for some $S \subseteq \mathbb{R}^{n}$. (E.g., $\pi_{\boldsymbol{y}}(\boldsymbol{x})=\langle\boldsymbol{y}, \boldsymbol{x}\rangle \boldsymbol{y} /\|\boldsymbol{y}\|^{2}$.) We write $S^{\perp}$ for the subspace of vectors orthogonal to $S$. For two additive subgroups $S_{1} \subseteq \mathbb{R}^{n}$ and $S_{2} \subseteq \mathbb{R}^{m}$, their direct sum $S_{1} \oplus S_{2} \subseteq \mathbb{R}^{n+m}$ is $\left\{(\boldsymbol{x}, \boldsymbol{y}): \boldsymbol{x} \in S_{1}, \boldsymbol{y} \in S_{2}\right\}$.

A convex body $K \subset \mathbb{R}^{n}$ is a convex compact subset of $\mathbb{R}^{n}$ with non-empty interior. It is symmetric if $-K=K$. A position of a convex body is simply $A K$ for a determinant-one matrix $A$. 


\subsection{Lattices}

A lattice $\mathcal{L} \subset \mathbb{R}^{n}$ of rank $d$ is the set of integer linear combinations of linearly independent basis vectors $\mathrm{B}:=\left(\boldsymbol{b}_{1}, \ldots, \boldsymbol{b}_{d}\right)$,

$$
\mathcal{L}=\mathcal{L}(\mathbf{B}):=\left\{\sum_{i=1}^{d} a_{i} \boldsymbol{b}_{i}: a_{i} \in \mathbb{Z}\right\} .
$$

We typically treat lattices as though they are full rank (i.e., $d=n$ ) by implicitly identifying $\operatorname{span}(\mathcal{L})$ with $\mathbb{R}^{d}$. The dual lattice

$$
\mathcal{L}^{*}:=\{\boldsymbol{w} \in \operatorname{span}(\mathcal{L}): \forall \boldsymbol{y} \in \mathcal{L},\langle\boldsymbol{w}, \boldsymbol{y}\rangle \in \mathbb{Z}\}
$$

is the set of all vectors in the span of $\mathcal{L}$ that have integer linear products with all lattice vectors. One can check that $\mathcal{L}^{* *}=\mathcal{L}$ and that $\mathcal{L}^{*}$ is generated by $\mathbf{B}^{*}:=\mathbf{B}\left(\mathbf{B}^{T} \mathbf{B}\right)^{-1}$.

We write

$$
\lambda_{1}(\mathcal{L}):=\min _{\boldsymbol{y} \in \mathcal{L} \backslash\{0\}}\|\boldsymbol{y}\|
$$

for the length of the shortest non-zero lattice vector. The covering radius is

$$
\mu(\mathcal{L}):=\max _{\boldsymbol{t} \in \operatorname{span}(\mathcal{L})} \min _{\boldsymbol{y} \in \mathcal{L}}\|\boldsymbol{t}-\boldsymbol{y}\| .
$$

The determinant of the lattice is given by $\operatorname{det}(\mathcal{L}):=\sqrt{\operatorname{det}\left(\mathbf{B}^{T} \mathbf{B}\right)}$, or simply $|\operatorname{det}(\mathbf{B})|$ in the full-rank case. One can show that the determinant is well defined (i.e., it does not depend on the choice of basis B). It follows that, if $\mathcal{L} \subset \mathbb{R}^{n}$ and $A \in \mathbb{R}^{n \times n}$ is non-singular, then $\operatorname{det}(A \mathcal{L})=|\operatorname{det}(A)| \operatorname{det}(\mathcal{L})$, and that $\operatorname{det}\left(\mathcal{L}^{*}\right)=1 / \operatorname{det}(\mathcal{L})$.

A sublattice $\mathcal{L}^{\prime} \subseteq \mathcal{L}$ is an additive subgroup of $\mathcal{L}$. We say that $\mathcal{L}^{\prime}$ is primitive if $\mathcal{L}^{\prime}=\mathcal{L} \cap \operatorname{span}\left(\mathcal{L}^{\prime}\right)$. For a primitive sublattice $\mathcal{L}^{\prime} \subseteq \mathcal{L}$, we define the quotient lattice $\mathcal{L} / \mathcal{L}^{\prime}:=\pi_{\mathcal{L}^{\prime \perp}}(\mathcal{L})$ to be the projection of $\mathcal{L}$ onto the space orthogonal to $\mathcal{L}^{\prime}$. In particular, $\mathcal{L} / \mathcal{L}^{\prime}$ is a lattice, and we have the identities $\left(\mathcal{L} / \mathcal{L}^{\prime}\right)^{*}=\mathcal{L}^{*} \cap \operatorname{span}\left(\mathcal{L}^{\prime}\right)^{\perp}$ and $\operatorname{det}\left(\mathcal{L} / \mathcal{L}^{\prime}\right)=\operatorname{det}(\mathcal{L}) / \operatorname{det}\left(\mathcal{L}^{\prime}\right)$.

For a parameter $s>0$ and $\boldsymbol{x} \in \mathbb{R}^{n}$, we define $\rho_{s}(\boldsymbol{x})=e^{-\pi\|\boldsymbol{x}\|^{2} / s^{2}}$. Then, for any discrete set $A$, we define its Gaussian mass as $\rho_{S}(A)=$ $\sum_{\boldsymbol{x} \in A} \rho_{s}(\boldsymbol{x})$. When $s=1$, we omit the subscript.

We recall the Poisson Summation Formula for the Gaussian mass of a lattice, which says that

$$
\rho_{s}(\mathcal{L})=\frac{s^{n}}{\operatorname{det}(\mathcal{L})} \cdot \rho_{1 / s}\left(\mathcal{L}^{*}\right)
$$

for any $s>0$ and (full-rank) lattice $\mathcal{L} \subset \mathbb{R}^{n}$.

Lemma 2.1 ([2, Lemma 1.5]). For any lattice $\mathcal{L} \subset \mathbb{R}^{n}$, shift vector $\boldsymbol{u} \in \mathbb{R}^{n}$, and any $r \geq 1 / \sqrt{2 \pi}$,

$$
\rho\left((\mathcal{L}-\boldsymbol{u}) \backslash r \sqrt{n} B_{2}^{n}\right) \leq\left(\sqrt{2 \pi e r^{2}} e^{-\pi r^{2}}\right)^{n} \cdot \rho(\mathcal{L}) .
$$

The following claim is an immediate consequence of the Poisson Summation Formula.

Claim 2.2. For any lattice $\mathcal{L} \subset \mathbb{R}^{n}$, shift vector $\boldsymbol{u} \in \mathbb{R}^{n}$, and parameter $s>0, \rho_{s}(\mathcal{L}-\boldsymbol{u}) \leq \rho_{s}(\mathcal{L})$ with equality if and only if $\boldsymbol{u} \in \mathcal{L}$.

Lemma 2.3. For any lattice $\mathcal{L} \subset \mathbb{R}^{n}$, primitive sublattice $\mathcal{L}^{\prime} \subset \mathcal{L}$, and $s>0$,

$$
\rho_{s}(\mathcal{L}) \leq \rho_{s}\left(\mathcal{L}^{\prime} \oplus \mathcal{L} / \mathcal{L}^{\prime}\right)=\rho_{s}\left(\mathcal{L}^{\prime}\right) \rho_{s}\left(\mathcal{L} / \mathcal{L}^{\prime}\right),
$$

with equality if and only if $\mathcal{L}=\mathcal{L}^{\prime} \oplus \mathcal{L} / \mathcal{L}^{\prime}$.

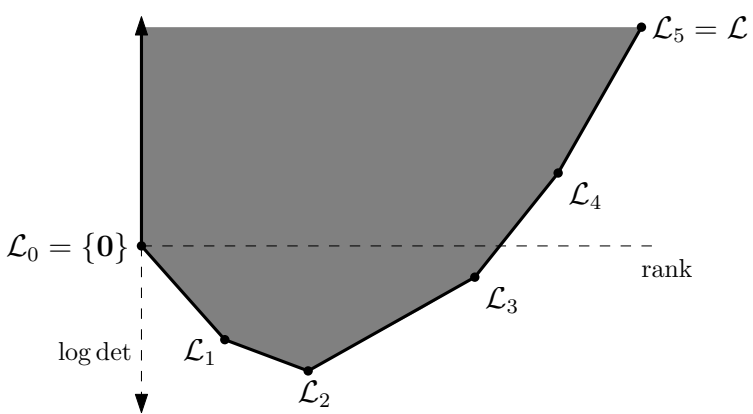

Figure 1: The canonical polygon of a (hypothetical) lattice $\mathcal{L}$.

Proof. Let $\widehat{C}:=\left\{\mathcal{L}^{\prime}+\boldsymbol{y}: \boldsymbol{y} \in \mathcal{L}\right\}$ be the set of distinct cosets of $\mathcal{L}^{\prime}$ over $\mathcal{L}, \pi:=\pi_{\text {span }\left(\mathcal{L}^{\prime}\right)}$, and $\pi^{\perp}:=\pi_{\text {span }\left(\mathcal{L}^{\prime}\right)^{\perp}}$. Then,

$$
\begin{aligned}
\sum_{\boldsymbol{y} \in \mathcal{L}} \rho_{s}(\boldsymbol{y}) & =\sum_{\boldsymbol{c} \in \widehat{C}} \rho_{s}(\boldsymbol{c}) \\
& =\sum_{\boldsymbol{c} \in \widehat{C}} \rho_{S}(\pi(\boldsymbol{c})) \rho_{s}\left(\pi^{\perp}(\boldsymbol{c})\right) \leq \rho_{s}\left(\mathcal{L}^{\prime}\right) \sum_{\boldsymbol{c} \in \widehat{C}} \rho_{s}\left(\pi^{\perp}(\boldsymbol{c})\right) \\
& =\rho_{S}\left(\mathcal{L}^{\prime}\right) \rho_{s}\left(\mathcal{L} / \mathcal{L}^{\prime}\right),
\end{aligned}
$$

where the inequality follows from Claim 2.2.

\subsection{Stability}

We say that a lattice $\mathcal{L} \subset \mathbb{R}^{n}$ is stable if $\operatorname{det}(\mathcal{L})=1$ and $\operatorname{det}\left(\mathcal{L}^{\prime}\right) \geq$ 1 for all sublattices $\mathcal{L}^{\prime} \subseteq \mathcal{L}$. (Some authors call such lattices "semistable.") Note the obvious relationship between this notion and Theorem 1.2. Here, we describe the properties of stable lattices that we will need in the sequel, and include proofs for completeness. This theory was developed by $[16,20,38]$. See, e.g., $[6,16]$ for a more thorough treatment.

We can in some sense "decompose" any lattice into stable lattices. To see this, we consider the two-dimensional scatter plot with points

$$
\left\{\left(\operatorname{rank}\left(\mathcal{L}^{\prime}\right), \log \operatorname{det}\left(\mathcal{L}^{\prime}\right)\right): \mathcal{L}^{\prime} \subseteq \mathcal{L}\right\},
$$

for some lattice $\mathcal{L} \subset \mathbb{R}^{n}$, where we explicitly include the trivial sublattice $\{0\}$ and define $\log \operatorname{det}(\{0\}):=0$. We call this the canonical plot of $\mathcal{L}$. Note that these points are bounded from below and that the minimum log det for each fixed rank is achieved. The convex hull of these points is therefore a degenerate polygon (bounded from below, but unbounded from above), called the canonical polygon of $\mathcal{L}$. See Figure 1.

We are interested in the vertices of this polygon (i.e., the extremal points), which correspond to certain primitive sublattices of $\mathcal{L}$ with low determinants. (E.g., $\mathcal{L}_{0}, \ldots, \mathcal{L}_{5}$ in Figure 1.) Each vertex corresponds to a unique sublattice, and a lattice $\mathcal{L}_{1}$ corresponding to a low-rank extremal point is a sublattice of any lattice $\mathcal{L}_{2}$ corresponding to a higher-rank extremal point, $\mathcal{L}_{1} \subset \mathcal{L}_{2}$. Therefore, the extremal points define a canonical filtration of $\mathcal{L}$,

$$
\{0\}=\mathcal{L}_{0} \subset \mathcal{L}_{1} \subset \cdots \subset \mathcal{L}_{k}=\mathcal{L} .
$$

(E.g., the canonical filtration of $\mathbb{Z}^{n}$ is trivial: $\{0\}=\mathcal{L}_{0} \subset \mathcal{L}_{1}=\mathbb{Z}^{n}$. Note in particular we only include lattices that correspond to vertices in the canonical filtration, not any lattice on the boundary.) All 
of the quotients $\mathcal{L}_{i} / \mathcal{L}_{i-1}$ of adjacent sublattices in the canonical filtration are scalings of stable lattices. This is what we mean when we say that we can "decompose" a lattice into a sequence of stable lattices. Following $[6,16]$, we make these facts (and more) precise in Proposition 2.5, which lists basic properties of the canonical filtration and stable lattices. We first need the following lemma, due to Stuhler [38].

Lemma 2.4. For any $\mathcal{L} \subset \mathbb{R}^{n}$ and any two primitive sublattices $\mathcal{L}_{1}, \mathcal{L}_{2} \subseteq \mathcal{L}$

$\operatorname{rank}\left(\mathcal{L}_{1}\right)+\operatorname{rank}\left(\mathcal{L}_{2}\right)=\operatorname{rank}\left(\mathcal{L}_{1} \cap \mathcal{L}_{2}\right)+\operatorname{rank}\left(\mathcal{L}_{1}+\mathcal{L}_{2}\right)$,

and

$$
\operatorname{det}\left(\mathcal{L}_{1} \cap \mathcal{L}_{2}\right) \operatorname{det}\left(\mathcal{L}_{1}+\mathcal{L}_{2}\right) \leq \operatorname{det}\left(\mathcal{L}_{1}\right) \operatorname{det}\left(\mathcal{L}_{2}\right),
$$

where we define $\operatorname{det}(\{0\})=1$.

Proof. The equality of ranks follows by considering the dimensions of the subspaces spanned by $\mathcal{L}_{1}, \mathcal{L}_{2}, \mathcal{L}_{1} \cap \mathcal{L}_{2}$, and $\mathcal{L}_{1}+\mathcal{L}_{2}$. For the inequality, suppose that $\mathcal{M}_{1}, \mathcal{M}_{2} \subseteq \mathcal{M}$ are sublattices such that $\mathcal{M}_{1} \cap \mathcal{M}_{2}=\{0\}$ and $\mathcal{M}_{1}+\mathcal{M}_{2}=\mathcal{M}$. Then, we have

$\operatorname{det}(\mathcal{M})=\operatorname{det}\left(\mathcal{M}_{1}\right) \cdot \operatorname{det}\left(\pi_{\mathrm{span}}\left(\mathcal{M}_{1}\right)^{\perp}\left(\mathcal{M}_{2}\right)\right) \leq \operatorname{det}\left(\mathcal{M}_{1}\right) \operatorname{det}\left(\mathcal{M}_{2}\right)$. Plugging in $\mathcal{M}:=\left(\mathcal{L}_{1}+\mathcal{L}_{2}\right) /\left(\mathcal{L}_{1} \cap \mathcal{L}_{2}\right), \mathcal{M}_{1}:=\mathcal{L}_{1} /\left(\mathcal{L}_{1} \cap \mathcal{L}_{2}\right)$ and $\mathcal{M}_{2}:=\mathcal{L}_{2} /\left(\mathcal{L}_{1} \cap \mathcal{L}_{2}\right)$ gives

$$
\begin{aligned}
\frac{\operatorname{det}\left(\mathcal{L}_{1}+\mathcal{L}_{2}\right)}{\operatorname{det}\left(\mathcal{L}_{1} \cap \mathcal{L}_{2}\right)} & =\operatorname{det}\left(\left(\mathcal{L}_{1}+\mathcal{L}_{2}\right) /\left(\mathcal{L}_{1} \cap \mathcal{L}_{2}\right)\right) \\
& \leq \operatorname{det}\left(\mathcal{L}_{1} /\left(\mathcal{L}_{1} \cap \mathcal{L}_{2}\right)\right) \operatorname{det}\left(\mathcal{L}_{2} /\left(\mathcal{L}_{1} \cap \mathcal{L}_{2}\right)\right) \\
& =\operatorname{det}\left(\mathcal{L}_{1}\right) \operatorname{det}\left(\mathcal{L}_{2}\right) / \operatorname{det}\left(\mathcal{L}_{1} \cap \mathcal{L}_{2}\right)^{2}
\end{aligned}
$$

The result follows by rearranging.

Proposition 2.5. For any lattice $\mathcal{L} \subset \mathbb{R}^{n}$, let the sequence of sublattices $\{0\}=\mathcal{L}_{0}, \mathcal{L}_{1}, \ldots, \mathcal{L}_{k}=\mathcal{L}$ be all sublattices corresponding to vertices of the canonical polytope, ordered by their rank. (See Figure 1.) Then,

(1) the $\mathcal{L}_{i}$ define a filtration $\mathcal{L}_{0} \subset \mathcal{L}_{1} \subset \cdots \subset \mathcal{L}_{k}$;

(2) the quotient lattice $\mathcal{L}_{i} / \mathcal{L}_{i-1}$ is a scaling of a stable lattice for $1 \leq i \leq k$ (i.e., $\operatorname{det}\left(\mathcal{L}_{i} / \mathcal{L}_{i-1}\right)^{-1 / \operatorname{rank}\left(\mathcal{L}_{i} / \mathcal{L}_{i-1}\right)} \cdot \mathcal{L}_{i} / \mathcal{L}_{i-1}$ is stable); and

(3) for all $1 \leq i \leq k-1, \operatorname{det}\left(\mathcal{L}_{i} / \mathcal{L}_{i-1}\right)^{1 / \operatorname{rank}\left(\mathcal{L}_{i} / \mathcal{L}_{i-1}\right)}<$ $\operatorname{det}\left(\mathcal{L}_{i+1} / \mathcal{L}_{i}\right)^{1 / \operatorname{rank}\left(\mathcal{L}_{i+1} / \mathcal{L}_{i}\right)}$.

Furthermore,

(i) the dual of a stable lattice is stable;

(ii) the set of all stable lattices is compact;

(iii) the direct sum of stable lattices is stable; and

(iv) a lattice $\mathcal{L} \subset \mathbb{R}^{n}$ is on the boundary of the set of stable lattices if and only if $\mathcal{L}$ is stable and there is a primitive sublattice $\mathcal{L}^{\prime} \subset \mathcal{L}$ with $0<\operatorname{rank}\left(\mathcal{L}^{\prime}\right)<n$ such that $\mathcal{L}^{\prime}$ and $\mathcal{L} / \mathcal{L}^{\prime}$ are both stable.

Proof. To prove Item 1 , we first note that for any two indices $i \leq j$, we can interpret Lemma 2.4 in terms of the canonical plot as follows. Consider the parallelogram with the three vertices $\left(\operatorname{rank}\left(\mathcal{L}_{i}\right), \log \operatorname{det}\left(\mathcal{L}_{i}\right)\right),\left(\operatorname{rank}\left(\mathcal{L}_{i}+\mathcal{L}_{j}\right), \log \operatorname{det}\left(\mathcal{L}_{i}+\mathcal{L}_{j}\right)\right)$, and $\left(\operatorname{rank}\left(\mathcal{L}_{i} \cap \mathcal{L}_{j}\right), \log \operatorname{det}\left(\mathcal{L}_{i} \cap \mathcal{L}_{j}\right)\right)$. Lemma 2.4 tells us that the point $\left(\operatorname{rank}\left(\mathcal{L}_{j}\right), \log \operatorname{det}\left(\mathcal{L}_{j}\right)\right)$ lies on or above the fourth point in this parallelogram. This contradicts the assumption that $\mathcal{L}_{i}$ and $\mathcal{L}_{j}$ are extremal points of the convex hull of the canonical plot unless the parallelogram is degenerate-i.e., unless $\mathcal{L}_{i}+\mathcal{L}_{j}=\mathcal{L}_{j}$ or $\mathcal{L}_{i} \cap \mathcal{L}_{j}=\mathcal{L}_{i}$. This happens if and only if $\mathcal{L}_{i} \subseteq \mathcal{L}_{j}$, as needed.

To prove Item 2 , let $\mathcal{L}^{\prime} \subseteq \mathcal{L}_{i} / \mathcal{L}_{i-1}$. Let $\widehat{\mathcal{L}} \subseteq \mathcal{L}_{i}$ be a "lift" of $\mathcal{L}^{\prime}$ so that $\mathcal{L}_{i-1} \subseteq \widehat{\mathcal{L}}$ and $\mathcal{L}^{\prime}=\widehat{\mathcal{L}} / \mathcal{L}_{i-1}$. Since $\mathcal{L}_{i-1}$ and $\mathcal{L}_{i}$ are vertices of the canonical polygon, the point $(\operatorname{rank}(\widehat{\mathcal{L}}), \log \operatorname{det}(\widehat{\mathcal{L}}))$ must lie on or above the line between $\left(\operatorname{rank}\left(\mathcal{L}_{i-1}\right), \log \operatorname{det}\left(\mathcal{L}_{i-1}\right)\right)$ and $\left(\operatorname{rank}\left(\mathcal{L}_{i}\right), \log \operatorname{det}\left(\mathcal{L}_{i}\right)\right)$. Therefore,

$$
\begin{aligned}
\operatorname{det}\left(\mathcal{L}^{\prime}\right) & =\operatorname{det}(\widehat{\mathcal{L}}) / \operatorname{det}\left(\mathcal{L}_{i-1}\right) \\
& \geq\left(\frac{\operatorname{det}\left(\mathcal{L}_{i}\right)}{\operatorname{det}\left(\mathcal{L}_{i-1}\right)}\right)^{\frac{\operatorname{rank}(\widehat{\mathcal{L}})-\operatorname{rank}\left(\mathcal{L}_{i-1}\right)}{\operatorname{rank}\left(\mathcal{L}_{i}\right)-\operatorname{rank}\left(\mathcal{L}_{i-1}\right)}} \\
& =\operatorname{det}\left(\mathcal{L}_{i} / \mathcal{L}_{i-1}\right)^{\frac{\operatorname{rank}\left(\mathcal{L}^{\prime}\right)}{\operatorname{rank}\left(\mathcal{L}_{i} / \mathcal{L}_{i-1}\right)}}
\end{aligned}
$$

I.e., if we set $\alpha_{i}:=\operatorname{det}\left(\mathcal{L}_{i} / \mathcal{L}_{i-1}\right)^{-1 / \operatorname{rank}\left(\mathcal{L}_{i} / \mathcal{L}_{i-1}\right)}$, then $\operatorname{det}\left(\alpha_{i} \mathcal{L}^{\prime}\right) \geq$ 1. It follows that $\alpha_{i} \mathcal{L}_{i} / \mathcal{L}_{i-1}$ is stable, as claimed.

Item 3 simply says that the slopes of the lines between vertices on the canonical polytope are strictly increasing. This is essentially just the definition of a vertex. (See Figure 1.)

To prove Item (i), let $\mathcal{M} \subset \mathbb{R}^{n}$ be a stable lattice and let $\mathcal{M}^{\prime} \subseteq$ $\mathcal{M}^{*}$ be a primitive sublattice of the dual. We have

$$
\begin{aligned}
\operatorname{det}\left(\mathcal{M}^{\prime}\right) & =\frac{1}{\operatorname{det}\left(\mathcal{M}^{*} / \mathcal{M}^{\prime}\right)} \\
& =\operatorname{det}\left(\left(\mathcal{M}^{*} / \mathcal{M}^{\prime}\right)^{*}\right) \\
& =\operatorname{det}\left(\mathcal{M} \cap \operatorname{span}\left(\mathcal{M}^{\prime}\right)\right) \\
& \geq 1 .
\end{aligned}
$$

Therefore, $\mathcal{M}^{*}$ is stable.

To prove Item (ii), it suffices to find a bounded set in $\mathbb{R}^{n \times n}$ that contains a basis for every stable lattice. Indeed, for any stable lattice $\mathcal{M} \subset \mathbb{R}^{n}$, by Item (i), we know that its dual $\mathcal{M}^{*}$ is also stable. Therefore, $\lambda_{1}\left(\mathcal{M}^{*}\right) \geq 1$. It then follows from [26] that there exists a basis $\left(\boldsymbol{b}_{1}, \ldots, \boldsymbol{b}_{n}\right)$ of $\mathcal{M}$ with $1 \leq\left\|\boldsymbol{b}_{i}\right\| \leq C n^{2.5}$ for all $i$, as needed.

To prove Item (iii), let $\mathcal{M}_{1}, \mathcal{M}_{2}$ be two stable lattices, and let $\mathcal{M}^{\prime} \subset \mathcal{M}_{1} \oplus \mathcal{M}_{2}$ be a sublattice. Then, applying Lemma 2.4, we have

$$
\begin{aligned}
\operatorname{det}\left(\mathcal{M}^{\prime}\right) & \geq \frac{\operatorname{det}\left(\mathcal{M}^{\prime} \cap \mathcal{M}_{1}\right) \operatorname{det}\left(\mathcal{M}^{\prime}+\mathcal{M}_{1}\right)}{\operatorname{det}\left(\mathcal{M}_{1}\right)} \\
& =\operatorname{det}\left(\mathcal{M}^{\prime} \cap \mathcal{M}_{1}\right) \operatorname{det}\left(\mathcal{M}^{\prime}+\mathcal{M}_{1}\right) .
\end{aligned}
$$

Note that $\mathcal{M}^{\prime} \cap \mathcal{M}_{1}$ is a sublattice of $\mathcal{M}_{1}$, so that $\operatorname{det}\left(\mathcal{M}^{\prime} \cap \mathcal{M}_{1}\right) \geq 1$. And $\mathcal{M}^{\prime}+\mathcal{M}_{1}=\mathcal{M}_{1} \oplus \pi_{\text {span }\left(\mathcal{M}_{2}\right)}\left(\mathcal{M}^{\prime}\right)$ is the direct sum of $\mathcal{M}_{1}$ with a sublattice of $\mathcal{M}_{2}$, so that $\operatorname{det}\left(\mathcal{M}^{\prime}+\mathcal{M}_{1}\right)=\operatorname{det}\left(\pi_{\operatorname{span}\left(\mathcal{M}_{2}\right)}\left(\mathcal{M}^{\prime}\right)\right) \geq$ 1 as well. The result follows.

Finally, Item (iv) follows by first noting that a stable lattice $\mathcal{M}$ is on the boundary if and only if there is some strict primitive non-zero sublattice $\mathcal{M}^{\prime} \subset \mathcal{M}$ with $\operatorname{det}\left(\mathcal{M}^{\prime}\right)=1$. Clearly, $\mathcal{M}^{\prime}$ is stable, since it has determinant one and all of its sublattices are also sublattices of $\mathcal{M}$, so that they must have determinant at least one. The proof that $\mathcal{M} / \mathcal{M}^{\prime}$ is stable is essentially identical to the proof of Item 2.

\subsection{The Voronoi Cell and Fundamental Bodies}

The Voronoi cell of a lattice $\mathcal{L} \subset \mathbb{R}^{n}$,

$$
\mathcal{V}(\mathcal{L}):=\left\{\boldsymbol{x} \in \mathbb{R}^{n}:\langle\boldsymbol{x}, \boldsymbol{y}\rangle \leq\|\boldsymbol{y}\|^{2} / 2, \forall \boldsymbol{y} \in \mathcal{L}\right\},
$$


is the set of vectors in $\mathbb{R}^{n}$ that are closer to 0 than to any other lattice vector. In fact, it is a symmetric polytope.

A fundamental body of a lattice $\mathcal{L} \subset \mathbb{R}^{n}$ is any convex body $K \subset \mathbb{R}^{n}$ such that $K+\mathcal{L}$ is a tiling of space. Equivalently, $\operatorname{vol}(K)=$ $\operatorname{det}(\mathcal{L})$ and $\operatorname{Int}(K) \cap(K+\boldsymbol{y})=\emptyset$ for any non-zero lattice point $\boldsymbol{y} \in \mathcal{L} \backslash\{0\}$. In particular, the Voronoi cell is a fundamental body.

CLAIM 2.6. For any lattice $\mathcal{L} \subset \mathbb{R}^{n}$, primitive sublattice $\mathcal{L}^{\prime} \subset \mathbb{R}^{n}$, fundamental body $K_{1}$ of $\mathcal{L}^{\prime}$, and fundamental body $K_{2}$ of $\mathcal{L} / \mathcal{L}^{\prime}$, $K:=K_{1} \times K_{2}$ is a fundamental body of $\mathcal{L}$. In particular, if $\{0\}=$ $\mathcal{L}_{0} \subset \mathcal{L}_{1} \subset \cdots \subset \mathcal{L}_{k}$ is a filtration of primitive sublattices, then

$$
\mathcal{V}\left(\bigoplus_{i} \mathcal{L}_{i} / \mathcal{L}_{i-1}\right)=\mathcal{V}\left(\mathcal{L}_{1} / \mathcal{L}_{0}\right) \times \cdots \times \mathcal{V}\left(\mathcal{L}_{k} / \mathcal{L}_{k-1}\right)
$$

is a fundamental body of $\mathcal{L}$.

Proof. Notice that

$$
\operatorname{vol}(K)=\operatorname{vol}\left(K_{1}\right) \cdot \operatorname{vol}\left(K_{2}\right)=\operatorname{det}\left(\mathcal{L}^{\prime}\right) \cdot \operatorname{det}\left(\mathcal{L} / \mathcal{L}^{\prime}\right)=\operatorname{det}(\mathcal{L}) .
$$

It therefore suffices to show that $\operatorname{Int}(K) \cap(K+\boldsymbol{y})=\emptyset$ for any $\boldsymbol{y} \in \mathcal{L} \backslash\{\mathbf{0}\}$. So suppose $\boldsymbol{y} \in \mathcal{L}$ such that $\operatorname{Int}(K) \cap(K+\boldsymbol{y}) \neq \emptyset$. Then, by projecting orthogonally to $\mathcal{L}^{\prime}$, we see that $\operatorname{Int}\left(K_{2}\right) \cap\left(K_{2}+\right.$

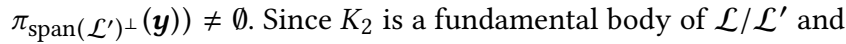

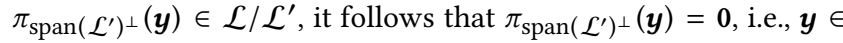
$\mathcal{L}^{\prime}$. Intersecting with $\operatorname{span}\left(\mathcal{L}^{\prime}\right)$, this implies that $\operatorname{Int}\left(K_{1}\right) \cap\left(K_{1}+\boldsymbol{y}\right) \neq$ $\emptyset$. Since $\boldsymbol{y} \in \mathcal{L}^{\prime}$ and $K_{1}$ is a fundamental body of $\mathcal{L}^{\prime}$, we obtain that $\boldsymbol{y}=\mathbf{0}$. The result follows.

The next lemma and its corollary show that the Voronoi cell is in some sense the "optimal fundamental body." They are very similar to some results due to Dadush [12, Lemma 6.3.6, Corollary 6.3.7].

Lemma 2.7. For any lattice $\mathcal{L} \subset \mathbb{R}^{n}$, there is a map $\psi_{\mathcal{L}}: \mathbb{R}^{n} \rightarrow$ $\mathcal{V}(\mathcal{L})$ such that $\left\|\psi_{\mathcal{L}}(\boldsymbol{x})\right\| \leq\|\boldsymbol{x}\|$, and for every fundamental body $K$ of $\mathcal{L}, \psi_{\mathcal{L}}$ restricted to $\operatorname{Int}(K)$ is injective and volume-preserving.

Proof. The function $\psi_{\mathcal{L}}$ just maps $x$ to the unique representative of $x \bmod \mathcal{L}$ that is in the Voronoi cell. Specifically, let $\mathrm{CVP}_{\mathcal{L}}(\boldsymbol{x}):=$ $\operatorname{argmin}_{\boldsymbol{y} \in \mathcal{L}}\|\boldsymbol{y}-\boldsymbol{x}\|$ be the closest lattice vector to $\boldsymbol{x}$, and let $\psi_{\mathcal{L}}(\boldsymbol{x}):=$ $\boldsymbol{x}-\mathrm{CVP}_{\mathcal{L}}(\boldsymbol{x})$. By the definition of CVP, it is immediate that $\left\|\psi_{\mathcal{L}}(\boldsymbol{x})\right\|=$ $\min _{\boldsymbol{y} \in \mathcal{L}}\|\boldsymbol{y}-\boldsymbol{x}\| \leq\|\boldsymbol{x}\|$.

Suppose $\psi_{\mathcal{L}}(\boldsymbol{x})=\psi_{\mathcal{L}}\left(\boldsymbol{x}^{\prime}\right)$ for some $\boldsymbol{x}, \boldsymbol{x}^{\prime} \in \operatorname{Int}(K)$. I.e., $\boldsymbol{x}-$ $\mathrm{CVP}_{\mathcal{L}}(x)=x^{\prime}-\operatorname{CVP}_{\mathcal{L}}\left(x^{\prime}\right)$. Rearranging, we see that $y:=x-x^{\prime}=$ $\mathrm{CVP}_{\mathcal{L}}(\boldsymbol{x})-\mathrm{CVP}_{\mathcal{L}}\left(\boldsymbol{x}^{\prime}\right)$ is a lattice point. But, $\boldsymbol{x} \in \operatorname{Int}(K) \cap(K+\boldsymbol{y})$. Since $K$ is a fundamental body, it follows that $\boldsymbol{y}=\mathbf{0}$. I.e., $\boldsymbol{x}=\boldsymbol{x}^{\prime}$, and $\psi_{\mathcal{L}}$ is injective over $\operatorname{Int}(K)$.

The fact that $\psi_{\mathcal{L}}$ is volume-preserving over $\operatorname{Int}(K)$ follows from the fact that it is an injective piecewise combination of translations.

COROLlary 2.8. For any non-decreasing measurable function $f$ : $\mathbb{R} \rightarrow \mathbb{R}$, lattice $\mathcal{L} \subset \mathbb{R}^{n}$, and fundamental body $K$ of $\mathcal{L}$,

$$
\int_{\mathcal{V}(\mathcal{L})} f(\|x\|) \mathrm{d} x \leq \int_{K} f(\|x\|) \mathrm{d} x .
$$

Proof.

$$
\begin{aligned}
\int_{K} f(\|x\|) \mathrm{d} x & =\int_{\operatorname{Int}(K)} f(\|x\|) \mathrm{d} \boldsymbol{x} \\
& \geq \int_{\operatorname{Int}(K)} f\left(\left\|\psi_{\mathcal{L}}(\boldsymbol{x})\right\|\right) \mathrm{d} \boldsymbol{x} \\
& =\int_{\psi_{\mathcal{L}}(\operatorname{Int}(K))} f(\|x\|) \mathrm{d} \boldsymbol{x} \\
& =\int_{\mathcal{V}(\mathcal{L})} f(\|x\|) \mathrm{d} \boldsymbol{x},
\end{aligned}
$$

where the last equality follows from the fact that $\psi_{\mathcal{L}}$ preserves volume and $\operatorname{vol}(\operatorname{Int}(K))=\operatorname{vol}(\mathcal{V}(\mathcal{L}))$, so it must be the case that $\psi_{\mathcal{L}}(\operatorname{Int}(K)) \subset \mathcal{V}(\mathcal{L})$ differs from $\mathcal{V}(\mathcal{L})$ on a set of measure zero.

\section{GRADIENTS OVER LATTICES AND OVER POSITIONS OF THE VORONOI CELL}

In the full version [34], we prove the following theorem.

THEOREM 3.1. For any continuously differentiable function $f$ : $\mathbb{R}^{\geq 0} \rightarrow \mathbb{R}$ and lattice $\mathcal{L} \subset \mathbb{R}^{n}$, let

$$
g(A):=\frac{1}{|\operatorname{det}(A)|} \cdot \int_{\mathcal{V}\left(A^{T} \mathcal{L}\right)} f\left(\|\boldsymbol{x}\|^{2}\right) \mathrm{d} \boldsymbol{x},
$$

and

$$
h(A):=\frac{1}{|\operatorname{det}(A)|} \cdot \int_{A \mathcal{V}(\mathcal{L})} f\left(\|\boldsymbol{x}\|^{2}\right) \mathrm{d} \boldsymbol{x},
$$

where $A \in \mathrm{GL}_{n}(\mathbb{R})$ ranges over the set of all non-singular matrices. Then, $g$ and $h$ are differentiable at $A=I_{n}$, with

$$
\left.\nabla_{A} g(A)\right|_{A=I_{n}}=\left.\nabla_{A} h(A)\right|_{A=I_{n}}=2 \int_{\mathcal{V}(\mathcal{L})} f^{\prime}\left(\|\boldsymbol{x}\|^{2}\right) \boldsymbol{x} \boldsymbol{x}^{T} \mathrm{~d} \boldsymbol{x},
$$

where $f^{\prime}(x):=\frac{\mathrm{d}}{\mathrm{d} x} f(x)$.

\section{PROOF OF THE REVERSE MINKOWSKI THEOREM}

In this section, we prove our main theorem, Theorem 1.2. Recall that the Voronoi cell $\mathcal{V}(\mathcal{L})$ of a lattice $\mathcal{L} \subset \mathbb{R}^{n}$ is the symmetric polytope of all vectors in $\mathbb{R}^{n}$ that are closer to 0 than to any other lattice vector,

$$
\mathcal{V}(\mathcal{L}):=\left\{\boldsymbol{x} \in \mathbb{R}^{n}: \forall \boldsymbol{y} \in \mathcal{L},\langle\boldsymbol{y}, \boldsymbol{x}\rangle \leq\|\boldsymbol{y}\|^{2} / 2\right\} .
$$

Also recall that for parameter $s>0, \gamma_{s}(\cdot)$ is the Gaussian measure on $\mathbb{R}^{n}$ given by

$$
\gamma_{S}(S):=\int_{S / s} e^{-\pi\|x\|^{2}} \mathrm{~d} \boldsymbol{x}
$$

for any measurable set $S \subseteq \mathbb{R}^{n}$. (Some authors prefer to parametrize $\gamma$ in terms of the standard deviation $\sigma:=s / \sqrt{2 \pi}$.) We are interested in the Gaussian mass $\gamma_{s}(\mathcal{V}(\mathcal{L}))$ of the Voronoi cell because, as the following lemma due to Chung, Dadush, Liu, and Peikert shows, this can be used to obtain an upper bound on the mass $\rho_{s}(\mathcal{L})$ of the lattice itself [7]. We include a proof for completeness.

Lemma 4.1 ([7, Lemma 3.4]). For any lattice $\mathcal{L} \subset \mathbb{R}^{n}$ and $s>0$,

$$
\rho_{s}(\mathcal{L}) \cdot \gamma_{S}(\mathcal{V}(\mathcal{L})) \leq 1 .
$$


Proof. By scaling appropriately, we may assume without loss of generality that $s=1$. Note that the Voronoi cell tiles space with respect to $\mathcal{L}$. I.e., $\bigcup_{\boldsymbol{y} \in \mathcal{L}}(\mathcal{V}(\mathcal{L})+\boldsymbol{y})=\mathbb{R}^{n}$, where the union is disjoint except on a measure-zero set. So,

$$
\begin{aligned}
1 & =\int_{\mathbb{R}^{n}} e^{-\pi\|\boldsymbol{x}\|^{2}} \mathrm{~d} \boldsymbol{x} \\
& =\sum_{\boldsymbol{y} \in \mathcal{L}} \int_{\mathcal{V}(\mathcal{L})} e^{-\pi\|\boldsymbol{y}+\boldsymbol{t}\|^{2}} \mathrm{~d} \boldsymbol{t} \\
& =\sum_{\boldsymbol{y} \in \mathcal{L}} e^{-\pi\|\boldsymbol{y}\|^{2}} \int_{\mathcal{V}(\mathcal{L})} e^{-\pi\|\boldsymbol{t}\|^{2}} e^{2 \pi\langle\boldsymbol{y}, \boldsymbol{t}\rangle} \mathrm{d} \boldsymbol{t} \\
& =\sum_{\boldsymbol{y} \in \mathcal{L}} \rho(\boldsymbol{y}) \int_{\mathcal{V}(\mathcal{L})} e^{-\pi\|\boldsymbol{t}\|^{2}} \cosh (2 \pi\langle\boldsymbol{y}, \boldsymbol{t}\rangle) \mathrm{d} \boldsymbol{t} \\
& \geq \sum_{\boldsymbol{y} \in \mathcal{L}} \rho(\boldsymbol{y}) \int_{\mathcal{V}(\mathcal{L})} e^{-\pi\|\boldsymbol{t}\|^{2}} \mathrm{~d} \boldsymbol{t} \\
& =\rho(\mathcal{L}) \gamma(\mathcal{V}(\mathcal{L})),
\end{aligned}
$$

where the fourth line follows from the fact that the Voronoi cell is symmetric.

Therefore, in order to prove Theorem 1.2, it suffices to show that $\gamma_{1 / t}(\mathcal{V}(\mathcal{L})) \geq 2 / 3$ for every lattice $\mathcal{L} \subset \mathbb{R}^{n}$ with $\operatorname{det}\left(\mathcal{L}^{\prime}\right) \geq 1$ for all sublattices $\mathcal{L}^{\prime} \subseteq \mathcal{L}$, where $t:=10(\log n+2)$. As we explained in the introduction, we will reduce this to studying local minima of the function $\mathcal{L} \mapsto \gamma_{1 / t}(\mathcal{V}(\mathcal{L}))$ over the set of determinant-one lattices. (We do not know whether such local minima actually exist.)

In Section 4.1, we collect some facts about the Gaussian mass of convex bodies. In Section 4.2, we apply these facts to the Voronoi cell to prove Theorem 1.2.

\subsection{Gaussian Mass of Convex Bodies}

We say that a measurable set $U \subset \mathbb{R}^{n}$ is in isotropic Gaussian position for parameter $s$ if

$$
\int_{U / s} e^{-\pi\|x\|^{2}} x x^{T} \mathrm{~d} x=\alpha \cdot I_{n}
$$

for some scalar $\alpha>0$. If $s=1$, we simply say that $U$ is in isotropic Gaussian position. Such a position has been considered elsewhere (e.g., [3]), but as far as we know, it did not previously have a name.

The main goal of this section is to prove the following theorem. We will also include a standard fact in Lemma 4.11 towards the end of this section.

Theorem 4.2. For any symmetric convex body $K \subset \mathbb{R}^{n}$ with $\operatorname{vol}(K) \geq 1$, if $K$ is in isotropic Gaussian position for some parameter $0<s \leq 1 / t$, then $\gamma_{s}(K) \geq 2 / 3$ where $t:=10(\log n+2)$.

Our proof of Theorem 4.2 proceeds in two parts. The first part is a result due to Bobkov [3] (Proposition 4.3 below), showing that an isotropic Gaussian position of a convex body has maximal Gaussian mass. We include a proof for completeness. In the second part (Theorem 4.6 below), we show that any volume-one convex body $K \subset \mathbb{R}^{n}$ has a position such that $\gamma_{s}(K) \geq 2 / 3$.

Proposition 4.3 ([3, Proposition 3.1]). For any symmetric convex body $K \subset \mathbb{R}^{n}$, if $K$ is in isotropic Gaussian position for some parameter $s>0$, then $\gamma_{s}(K) \geq \gamma_{s}(A K)$ for any determinant-one $\operatorname{matrix} A \in \mathrm{SL}_{n}(\mathbb{R})$.
We start by observing that isotropic Gaussian positions correspond to critical points of the Gaussian mass function over positions.

FACT 4.4. For any measurable set $U \subset \mathbb{R}^{n}$, let

$$
h(A):=\frac{\gamma(A U)}{|\operatorname{det}(A)|},
$$

where $A \in \mathrm{GL}_{n}(\mathbb{R})$ ranges over the non-singular matrices. Then,

$$
\left.\nabla_{A} h(A)\right|_{A=I_{n}}=-2 \pi \int_{U} e^{-\pi\|\boldsymbol{x}\|^{2}} \boldsymbol{x} \boldsymbol{x}^{T} \mathrm{~d} \boldsymbol{x} .
$$

In particular, $A \mapsto \gamma(A U)$ has a critical point at $I_{n}$ when restricted to determinant-one matrices if and only if $U$ is in isotropic Gaussian position.

We will also need the following result due to Cordero-Erausquin, Fradelizi, and Maurey [10], which is related to the so-called (B) conjecture due to Banaszczyk (see [27]).

TheOREM 4.5 ([10]). For any symmetric convex body $K \subset \mathbb{R}^{n}$, the function $\gamma\left(e^{D} K\right)$, where $D \in \mathbb{R}^{n \times n}$ ranges over all diagonal matrices, is log-concave.

Proof of Proposition 4.3. By scaling $K$, we may assume that $s=1$. Let $A=U D V$ be the singular-value decomposition of $A$. (I.e., $D$ is a diagonal matrix and $U$ and $V$ are orthogonal matrices.) Note that the Gaussian measure is invariant under orthogonal transformations, so that $\gamma(A K)=\gamma(U D V K)=\gamma(D V K)$. Let $K^{\prime}:=$ $V K$, and note that $\gamma\left(K^{\prime}\right)=\gamma(K)$ and that $K^{\prime}$ is in isotropic Gaussian position, since $V$ is an orthogonal transformation.

Let $\widehat{h}(M):=\gamma\left(e^{M} K^{\prime}\right) /\left|\operatorname{det}\left(e^{M}\right)\right|$. By Fact 4.4 and the chain rule, we have

$$
\left.\nabla_{M} \widehat{h}(M)\right|_{M=0}=-2 \pi \int_{K^{\prime}} e^{-\pi\|x\|^{2}} x x^{T} \mathrm{~d} \boldsymbol{x}=-\alpha \cdot I_{n}
$$

for some scalar $\alpha \in \mathbb{R}$, where the second equality is simply the fact that $K^{\prime}$ is in isotropic Gaussian position. Let $X \subset \mathbb{R}^{n \times n}$ be the set of trace-zero diagonal matrices. Then, the function $\widehat{h}_{X}$ obtained by restricting $\widehat{h}$ to $X$ has a critical point at zero, since $\operatorname{Tr}\left(I_{n} M\right)=0$ for any $M \in X$. By Theorem $4.5, \widehat{h}_{X}$ is log-concave, so that this critical point must be a global maximum. Therefore, $\gamma(A K)=\gamma\left(D K^{\prime}\right) \leq$ $\gamma\left(K^{\prime}\right)=\gamma(K)$, as needed.

We now proceed to the second part of the proof of Theorem 4.2.

TheORem 4.6. For any symmetric convex body $K \subset \mathbb{R}^{n}$ with volume one, there is a determinant-one matrix $A \in \mathrm{SL}_{n}(\mathbb{R})$ such that $\gamma_{1 / t}(A K) \geq 2 / 3$, where $t:=2 \sqrt{3 e}\left(\log _{2} n+2\right)<10(\log n+2)$.

The proof is based on an important theorem that follows from the work of Figiel and Tomczak-Jaegermann [15], Lewis [28], and Pisier [33]. We first need some definitions. Recall that any symmetric convex body $K \subset \mathbb{R}^{n}$ defines a norm $\|\cdot\|_{K}$ given by

$$
\|x\|_{K}:=\inf \{s \geq 0: x \in s K\} .
$$

We then define the $\ell$-norm on $\mathbb{R}^{n \times n}$ by

$$
\ell_{K}(A):=\left(\int_{\mathbb{R}^{n}}\|A x\|_{K}^{2} \mathrm{~d} \gamma(\boldsymbol{x})\right)^{1 / 2},
$$


where $\mathrm{d} \gamma(\boldsymbol{x}):=e^{-\pi\|\boldsymbol{x}\|^{2}} \mathrm{~d} \boldsymbol{x}$. Finally, we recall that a convex body $K$ has a polar given by

$$
K^{\circ}:=\left\{\boldsymbol{x} \in \mathbb{R}^{n}: \forall \boldsymbol{y} \in K,\langle\boldsymbol{y}, \boldsymbol{x}\rangle \leq 1\right\},
$$

which is itself a convex body.

Theorem 4.7 ([15, 28, 33]; See [12, Theorem 4.4.3]). For any symmetric convex body $K \subset \mathbb{R}^{n}$, there exists a determinant-one matrix $A \in \mathrm{SL}_{n}(\mathbb{R})$ such that

$$
\ell_{K}(A) \ell_{K^{\circ}}\left(A^{-T}\right) \leq n\left(\log _{2} n+2\right) / \pi .
$$

Lemma 4.8. For any symmetric convex body $K \subset \mathbb{R}^{n}$ with volume one and any determinant-one matrix $A \in \mathrm{SL}_{n}(\mathbb{R})$, we have

$$
\ell_{K^{\circ}}\left(A^{-T}\right) \geq \sqrt{n /(2 \pi)} \cdot r_{n}>n /(2 \pi \sqrt{e}) .
$$

where $_{n}:=\operatorname{vol}\left(B_{2}^{n}\right)^{-1 / n}>\sqrt{n /(2 \pi e)}$ is the radius such that $\operatorname{vol}\left(r_{n} B_{2}^{n}\right)=$ 1.

Proof. By replacing $K$ with $A K$, we may assume without loss of generality that $A=I_{n}$. Unpacking the definitions, we see that

$$
\begin{aligned}
\ell_{K^{\circ}}\left(I_{n}\right)^{2} & =\int_{\mathbb{R}^{n}}\|\boldsymbol{x}\|_{K^{\circ}}^{2} \mathrm{~d} \gamma(\boldsymbol{x}) \\
& =\int_{\mathbb{R}^{n}} \sup _{\boldsymbol{y} \in K}\langle\boldsymbol{y}, \boldsymbol{x}\rangle^{2} \mathrm{~d} \gamma(\boldsymbol{x}) \\
& =\left(\int_{\mathbb{R}^{n}}\|\boldsymbol{x}\|^{2} \mathrm{~d} \gamma(\boldsymbol{x})\right) \cdot\left(\int_{\mathbb{R}^{n}} \sup _{\boldsymbol{y} \in K} \frac{\langle\boldsymbol{y}, \boldsymbol{x}\rangle^{2}}{\|\boldsymbol{x}\|^{2}} \mathrm{~d} \gamma(\boldsymbol{x})\right) \\
& =\frac{n}{2 \pi} \cdot \int_{\mathbb{R}^{n}} \sup _{\boldsymbol{y} \in K} \frac{\langle\boldsymbol{y}, \boldsymbol{x}\rangle^{2}}{\|\boldsymbol{x}\|^{2}} \mathrm{~d} \gamma(\boldsymbol{x}),
\end{aligned}
$$

where we have used the fact that the Gaussian is spherically symmetric. By Jensen's inequality, we have

$$
\int_{\mathbb{R}^{n}} \sup _{\boldsymbol{y} \in K} \frac{\langle\boldsymbol{y}, \boldsymbol{x}\rangle^{2}}{\|\boldsymbol{x}\|^{2}} \mathrm{~d} \gamma(\boldsymbol{x}) \geq\left(\int_{\mathbb{R}^{n}} \sup _{\boldsymbol{y} \in K} \frac{\langle\boldsymbol{y}, \boldsymbol{x}\rangle}{\|\boldsymbol{x}\|} \mathrm{d} \gamma(\boldsymbol{x})\right)^{2},
$$

and by Urysohn's inequality (see [1, Theorem 1.5.11]), we have

$$
\int_{\mathbb{R}^{n}} \sup _{\boldsymbol{y} \in K} \frac{\langle\boldsymbol{y}, \boldsymbol{x}\rangle}{\|\boldsymbol{x}\|} \mathrm{d} \gamma(\boldsymbol{x}) \geq r_{n} .
$$

The result follows by combining everything together.

The following corollary is an immediate consequence of the previous two results.

COROLlary 4.9. For any symmetric convex body $K \subset \mathbb{R}^{n}$ with volume one, there exists a determinant-one matrix $A \in \mathrm{SL}_{n}(\mathbb{R})$ such that $\ell_{K}(A) \leq 2 \sqrt{e}\left(\log _{2} n+2\right)$.

Proof of Theorem 4.6. By Corollary 4.9, there exists an $A \in$ $\mathrm{SL}_{n}(\mathbb{R})$ such that

$$
\ell_{K}(A)^{2}=\int_{\mathbb{R}^{n}}\|A \boldsymbol{x}\|_{K}^{2} \mathrm{~d} \gamma(\boldsymbol{x}) \leq\left(2 \sqrt{e}\left(\log _{2} n+2\right)\right)^{2} .
$$

We will use $A^{-1}$ as our matrix. Note that

$$
\begin{aligned}
\gamma_{1 / t}\left(A^{-1} K\right) & =\int_{\mathbb{R}^{n}} 1_{\|\boldsymbol{x}\|_{A^{-1} K} \leq t} \mathrm{~d} \gamma(\boldsymbol{x}) \\
& =\int_{\mathbb{R}^{n}} 1_{\|A \boldsymbol{x}\|_{K} \leq t} \mathrm{~d} \gamma(\boldsymbol{x}) \\
& =1-\int_{\mathbb{R}^{n}} 1_{\|A \boldsymbol{x}\|_{K}>t} \mathrm{~d} \gamma(\boldsymbol{x}) .
\end{aligned}
$$

The result then follows by Markov's inequality, which tells us that

$$
\begin{aligned}
\int_{\mathbb{R}^{n}} 1_{\|A \boldsymbol{x}\|_{K}>t} \mathrm{~d} \gamma(\boldsymbol{x}) & \leq \frac{1}{t^{2}} \cdot \int_{\mathbb{R}^{n}}\|A \boldsymbol{x}\|_{K}^{2} \mathrm{~d} \gamma(\boldsymbol{x}) \\
& \leq \frac{1}{t^{2}} \cdot\left(2 \sqrt{e}\left(\log _{2} n+2\right)\right)^{2} \\
& =\frac{1}{3} .
\end{aligned}
$$

We now obtain Theorem 4.2 as an immediate corollary of Proposition 4.3 and Theorem 4.6.

Proof of Theorem 4.2. By Theorem 4.6, there is some $A \in$ $\mathrm{SL}_{n}(\mathbb{R})$ such that $\gamma_{s}(A K) \geq 2 / 3$, and by Proposition $4.3, \gamma_{s}(K) \geq$ $\gamma_{s}(A K) \geq 2 / 3$, as needed.

Concentration of Measure. We will also need a standard lemma about the concentration of Gaussian measure. We first recall the Gaussian isoperimetric inequality [4, 39] (see also [1, Theroem 3.1.9]).

Theorem 4.10 (Gaussian isoperimetric ineduality). For any measurable set $U \subset \mathbb{R}^{n}$ and any $\tau>0$,

$$
\gamma\left(U+\tau B_{2}^{n}\right) \geq \int_{-\infty}^{\sigma+\tau} e^{-\pi x^{2}} \mathrm{~d} x
$$

where $\sigma \in[-\infty, \infty]$ is such that $\int_{-\infty}^{\sigma} e^{-\pi x^{2}} \mathrm{~d} x=\gamma(U)$.

Recall that the inradius of a convex body $K$ is defined as $\max \{r \geq$ $0: r B_{2}^{n} \subseteq K$ \}, i.e., the radius of the largest ball contained in the body.

Lemma 4.11. If $K \subset \mathbb{R}^{n}$ is a convex body with $\gamma_{1 / t}(K) \geq 2 / 3$ for some $t>0$, then

$$
\gamma_{1 /(t+\tau)}(K) \geq 1-e^{-\pi r^{2} \tau^{2}} / 3,
$$

for any $\tau \geq 0$, where $r \geq 0$ is the inradius of $K$.

Proof. Note that

$$
\gamma_{1 /(t+\tau)}(K)=\gamma((t+\tau) K) \geq \gamma\left(t K+r \tau B_{2}^{n}\right),
$$

since by definition $r B_{2}^{n} \subseteq K$. Applying Theorem 4.10, we have

$$
\gamma_{1 /(t+\tau)}(K) \geq \int_{-\infty}^{\sigma+r \tau} e^{-\pi x^{2}} \mathrm{~d} x
$$

where $\sigma>0$ is such that $\int_{-\infty}^{\sigma} e^{-\pi x^{2}} \mathrm{~d} x=2 / 3$. Finally, we note that

$$
\begin{aligned}
\int_{-\infty}^{\sigma+r \tau} e^{-\pi r^{2}} \mathrm{~d} x & =1-\int_{\sigma+r \tau}^{\infty} e^{-\pi x^{2}} \mathrm{~d} x \\
& \geq 1-e^{-\pi r^{2} \tau^{2}} \int_{\sigma}^{\infty} e^{-\pi x^{2}} \mathrm{~d} x \\
& =1-e^{-\pi r^{2} \tau^{2}} / 3,
\end{aligned}
$$

where the inequality follows from the fact that $\sigma, \tau$, and $r$ are nonnegative, so that $(x+r \tau)^{2} \geq x^{2}+r^{2} \tau^{2}$ for all $x \geq \sigma$. The result follows. 


\subsection{Proof of Theorem 1.2}

We now use Theorem 3.1 and Theorem 4.2 to characterize local minima of $\gamma_{s}(\mathcal{V}(\mathcal{L}))$.

THEOREM 4.12. If $\mathcal{L} \subset \mathbb{R}^{n}$ corresponds to a local minimum (or maximum) of $\gamma_{1 / t}(\mathcal{V}(\mathcal{L}))$ over the set of determinant-one lattices, then $\mathcal{V}(\mathcal{L})$ is in isotropic Gaussian position with parameter $1 / t$, and

$$
\gamma_{1 / t}(\mathcal{V}(\mathcal{L})) \geq 2 / 3
$$

where $t:=10(\log n+2)$.

Proof. By Theorem 3.1 with $f(x)=t^{n} \cdot e^{-\pi t^{2} x}$, we have

$$
\begin{aligned}
\left.\nabla_{A}\left(\frac{\gamma_{1 / t}\left(\mathcal{V}\left(A^{T} \mathcal{L}\right)\right)}{|\operatorname{det}(A)|}\right)\right|_{A=I_{n}} & =2 \int_{\mathcal{V}(\mathcal{L})} f^{\prime}\left(\|\boldsymbol{x}\|^{2}\right) \boldsymbol{x} \boldsymbol{x}^{T} \mathrm{~d} \boldsymbol{x} \\
& =-2 \pi t^{n+2} \cdot \int_{\mathcal{V}(\mathcal{L})} \rho_{1 / t}(\boldsymbol{x}) \boldsymbol{x} \boldsymbol{x}^{T} \mathrm{~d} \boldsymbol{x} .
\end{aligned}
$$

Recall that $I_{n}$ corresponds to a local extremum of a differentiable function $g(A)$ restricted to the manifold of determinant-one matrices only if $\left.\nabla_{A} g(A)\right|_{A=I_{n}}$ is a scalar multiple of the identity. So, the above expression must be a multiple of the identity. I.e., $\mathcal{V}(\mathcal{L})$ is in isotropic Gaussian position. The result then follows from Theorem 4.2 .

Before moving to the proof of our main theorem, we need the following claim.

Claim 4.13. For any $x>1$,

$$
e^{-2 \log ^{2} x}+e^{-2 \log ^{2}(x /(x-1))}<1 .
$$

Proof. By symmetry, we may assume that $x \geq 2$. (Otherwise, we can replace $x$ with $x /(x-1)$.) If $2 \leq x \leq 2.5$, then

$$
e^{-2 \log ^{2} x}+e^{-2 \log ^{2}(x /(x-1))}<e^{-2 \log ^{2} 2}+e^{-2 \log ^{2}(5 / 3)}<1 .
$$

A similar computation works if $2.5 \leq x \leq e$. Finally, using the fact that $\log (x /(x-1))=-\log (1-1 / x)>1 / x$ for $x>1$, we have for any $x \geq e$ that

$$
e^{-2 \log ^{2} x}+e^{-2 \log ^{2}(x /(x-1))}<\frac{1}{x^{2}}+e^{-2 / x^{2}}<\frac{1}{x^{2}}+1-\frac{1}{x^{2}}=1 .
$$

We now prove our main theorem in the special case when $\mathcal{L}$ is a stable lattice. The full result will follow as a relatively straightforward corollary.

Proposition 4.14. For any stable lattice $\mathcal{L} \subset \mathbb{R}^{n}, \rho_{1 / t}(\mathcal{L}) \leq \frac{3}{2}$, where $t:=10(\log n+2)$.

Proof. By Lemma 4.1, it suffices to show that $\gamma_{1 / t}(\mathcal{V}(\mathcal{L})) \geq$ $2 / 3$. We assume for induction that $\gamma_{1 /(10(\log d+2))}\left(\mathcal{V}\left(\mathcal{L}^{\prime}\right)\right) \geq 2 / 3$ for any stable lattice $\mathcal{L}^{\prime}$ of rank $d<n$. (A quick check shows that this is true for $d=1$.) Since the set of stable lattices is compact by Item (ii) of Proposition 2.5 and the function $\gamma_{1 / t}(\mathcal{V}(\mathcal{L}))$ is continuous, we may assume without loss of generality that $\mathcal{L}$ corresponds to a global minimum of $\gamma_{1 / t}(\mathcal{V}(\mathcal{L}))$ over the set of stable lattices. If this global minimum is also a local minimum over the set of determinantone lattices, then by Theorem $4.12, \gamma_{1 / t}(\mathcal{V}(\mathcal{L})) \geq 2 / 3$, and we are done.

Otherwise, $\mathcal{L}$ lies on the boundary of the set of stable lattices. I.e., there is some primitive sublattice $\mathcal{L}^{\prime} \subset \mathcal{L}$ of rank $d<n$ such that $\mathcal{L}^{\prime}$ and $\mathcal{L} / \mathcal{L}^{\prime}$ are stable. (See Item (iv) of Proposition 2.5.) By Corollary 2.8 together with Claim 2.6, we have

$$
\begin{aligned}
\gamma_{1 / t}(\mathcal{V}(\mathcal{L})) & \geq \gamma_{1 / t}\left(\mathcal{V}\left(\mathcal{L} / \mathcal{L}^{\prime} \oplus \mathcal{L}^{\prime}\right)\right) \\
& =\gamma_{1 / t}\left(\mathcal{V}\left(\mathcal{L} / \mathcal{L}^{\prime}\right)\right) \cdot \gamma_{1 / t}\left(\mathcal{V}\left(\mathcal{L}^{\prime}\right)\right) .
\end{aligned}
$$

Let $t_{1}:=10(\log d+2)$ and $t_{2}:=10(\log (n-d)+2)$. By the induction hypothesis, we see that $\gamma_{1 / t_{1}}\left(\mathcal{V}\left(\mathcal{L}^{\prime}\right)\right) \geq 2 / 3$ and $\gamma_{1 / t_{2}}\left(\mathcal{V}\left(\mathcal{L} / \mathcal{L}^{\prime}\right)\right) \geq$ $2 / 3$. By Lemma 4.11, we therefore have

$$
\gamma_{1 / t}\left(\mathcal{V}\left(\mathcal{L}^{\prime}\right)\right) \geq 1-\frac{1}{3} \cdot e^{-2 \log ^{2}(n / d)},
$$

and

$$
\gamma_{1 / t}\left(\mathcal{V}\left(\mathcal{L} / \mathcal{L}^{\prime}\right)\right) \geq 1-\frac{1}{3} \cdot e^{-2 \log ^{2}(n /(n-d))},
$$

where we have used the fact that the inradius of the Voronoi cell is $\lambda_{1}(\mathcal{L}) / 2$, which is at least $1 / 2$ for a stable lattice (and the constant in the exponent is very loose). Therefore, using (4),

$$
\begin{aligned}
\gamma_{1 / t}(\mathcal{V}(\mathcal{L})) & \geq\left(1-\frac{1}{3} e^{-2 \log ^{2}(n / d)}\right)\left(1-\frac{1}{3} e^{-2 \log ^{2}(n /(n-d))}\right) \\
& >1-\frac{1}{3} \cdot\left(e^{-2 \log ^{2}(n / d)}+e^{-2 \log ^{2}(n /(n-d))}\right) \\
& >\frac{2}{3},
\end{aligned}
$$

where the last inequality follows from Claim 4.13 with $x:=n / d$.

So, for every stable lattice $\mathcal{L}$, we have $\gamma_{1 / t}(\mathcal{V}(\mathcal{L})) \geq 2 / 3$, and the result then follows from Lemma 4.1.

We now derive our main theorem as a corollary.

Proof of Theorem 1.2. Let $\{0\}=\mathcal{L}_{0} \subset \cdots \subset \mathcal{L}_{k}=\mathcal{L}$ be the canonical filtration of $\mathcal{L}$. Recall from Item 2 of Proposition 2.5 that with $\alpha_{i}:=\operatorname{det}\left(\mathcal{L}_{i} / \mathcal{L}_{i-1}\right)^{-1 / \operatorname{rank}\left(\mathcal{L}_{i} / \mathcal{L}_{i-1}\right)} \leq 1, \alpha_{i} \cdot\left(\mathcal{L}_{i} / \mathcal{L}_{i-1}\right)$ is a stable lattice. Therefore, by Lemma 2.3,

$$
\rho_{1 / t}(\mathcal{L}) \leq \rho_{1 / t}\left(\bigoplus_{i=1}^{k} \mathcal{L}_{i} / \mathcal{L}_{i-1}\right) \leq \rho_{1 / t}\left(\bigoplus_{i=1}^{k} \alpha_{i}\left(\mathcal{L}_{i} / \mathcal{L}_{i-1}\right)\right) .
$$

By Item (iii) of Proposition 2.5, this direct sum of stable lattices is itself a stable lattice. The result then follows from Proposition 4.14.

\section{PROOF OF THE COVERING RADIUS APPROXIMATION}

We will need the following lemma, which is implicit in [2]. $3 / 2$,

Lemma 5.1. For any lattice $\mathcal{L} \subset \mathbb{R}^{n}$ and $t>0$ such that $\rho_{1 / t}\left(\mathcal{L}^{*}\right) \leq$

$$
\mu(\mathcal{L})<\left(\sqrt{\frac{n}{2 \pi}}+1\right) \cdot t .
$$

Proof. By scaling the lattice, we may assume without loss of generality that $t=1$. Let $r:=((1+4 / \sqrt{n}) /(2 \pi))^{1 / 2}$. By Lemma 2.1, for any $t \in \mathbb{R}^{n}$, we have

$$
\begin{aligned}
\rho\left((\mathcal{L}-\boldsymbol{t}) \backslash r \sqrt{n} B_{2}^{n}\right) & \leq\left(\sqrt{2 \pi e r^{2}} e^{-\pi r^{2}}\right)^{n} \cdot \rho(\mathcal{L}) \\
& =e^{-2 \sqrt{n}}(1+4 / \sqrt{n})^{n / 2} \cdot \rho(\mathcal{L}) \\
& <\rho(\mathcal{L}) / 3,
\end{aligned}
$$

where the last inequality follows by noting that it holds for $n=1$ and that $e^{-2 x} \cdot(1+4 / x)^{x^{2} / 2}$ is a decreasing function in $x$ for $x>$ 
0 (a fact that can be proven using a tedious but straightforward computation). On the other hand, it is an easy consequence of the Poisson Summation Formula (see, e.g., [30]) that for any $t \in \mathbb{R}^{n}$,

$$
\rho(\mathcal{L}-\boldsymbol{t}) \geq \frac{2-\rho\left(\mathcal{L}^{*}\right)}{\rho\left(\mathcal{L}^{*}\right)} \cdot \rho(\mathcal{L}) \geq \rho(\mathcal{L}) / 3 .
$$

Therefore, $(\mathcal{L}-\boldsymbol{t}) \cap r \sqrt{n} B_{2}^{n}$ is nonempty, and in particular, $\operatorname{dist}(\boldsymbol{t}, \mathcal{L}) \leq$ $r \sqrt{n}<\sqrt{n /(2 \pi)}+1$. Since this holds for arbitrary $\boldsymbol{t}$, we have $\mu(\mathcal{L})<\sqrt{n /(2 \pi)}+1$, as needed.

We now note that Theorem 1.2 (together with Lemma 5.1) immediately implies a bound on the covering radius of stable lattices.

Theorem 5.2. For any stable lattice $\mathcal{L} \subset \mathbb{R}^{n}$,

$$
\mu(\mathcal{L}) \leq 4 \sqrt{n}(\log n+10) .
$$

Proof. Let $t:=10(\log n+2)$. Since $\mathcal{L}^{*}$ is also stable (by Item (i) of Proposition 2.5), Theorem 1.2 implies that $\rho_{1 / t}\left(\mathcal{L}^{*}\right) \leq 3 / 2$. Applying Lemma 5.1, we have

$$
\mu(\mathcal{L}) \leq(\sqrt{n /(2 \pi)}+1) \cdot t<4 \sqrt{n}(\log n+10),
$$

as needed.

Next, we show (Proposition 5.4) how to reduce the case of general lattices to the stable case. We will need the following technical lemma, which is a slight modification of [13, Lemma 4.9] (with an essentially identical proof).

LeMmA 5.3 (Reverse AM-GM). Let $0<a_{1}<\cdots<a_{k}$ and $d_{1}, \ldots, d_{k} \in \mathbb{N}$, and for $j=1, \ldots, k$, define $m_{j}:=\sum_{i \geq j} d_{i}$. Then,

$$
\sum_{i=1}^{k} d_{i} a_{i} \leq 2 e \cdot\left\lceil\log \left(2 m_{1}\right)\right\rceil \cdot \max _{j} m_{j}\left(\prod_{i \geq j} a_{i}^{d_{i}}\right)^{1 / m_{j}} .
$$

Proof. For $\ell=1,2, \ldots$, let $S_{\ell}:=\left\{j: e^{-\ell} a_{k}<a_{j} \leq e^{1-\ell} a_{k}\right\}$, and let $j_{\ell}:=\min \left\{j \in S_{\ell}\right\}$. For non-empty $S_{\ell}$, we have

$$
\sum_{i \in S_{\ell}} d_{i} a_{i} \leq m_{j_{\ell}} e^{1-\ell} a_{k} \leq e m_{j_{\ell}} a_{j_{\ell}} \leq e m_{j_{\ell}} \cdot\left(\prod_{i \geq j_{\ell}} a_{i}^{d_{i}}\right)^{1 / m_{j_{\ell}}} .
$$

Let $\ell^{*}:=\left\lceil\log \left(2 m_{1}\right)\right\rceil$. By the above inequality, it suffices to argue that there exists an $\ell$ such that $2 \ell^{*} \cdot \sum_{S_{\ell}} d_{i} a_{i} \geq \sum_{i=1}^{k} d_{i} a_{i}$. Indeed,

$$
\begin{aligned}
\sum_{\ell=1}^{\ell^{*}} \sum_{i \in S_{\ell}} d_{i} a_{i} & =\sum_{i=1}^{k} d_{i} a_{i}-\sum_{\ell>\ell^{*}} \sum_{i \in S_{\ell}} d_{i} a_{i} \\
& >\sum_{i=1}^{k} d_{i} a_{i}-m_{1} \cdot \frac{a_{k}}{2 m_{1}} \\
& \geq \frac{1}{2} \cdot \sum_{i=1}^{k} d_{i} a_{i},
\end{aligned}
$$

where in the last inequality we have used that $d_{k} \geq 1$. Therefore, there exists an $\ell$ such that

$$
\sum_{i \in S_{\ell}} d_{i} a_{i} \geq \frac{1}{2 \ell^{*}} \cdot \sum_{i=1}^{k} d_{i} a_{i}
$$

as needed.
Recall that

$$
\mu_{\operatorname{det}}(\mathcal{L}):=\max _{W \subset \mathbb{R}^{n}} \sqrt{\operatorname{dim}\left(W^{\perp}\right)} \cdot \operatorname{det}\left(\pi_{W^{\perp}}(\mathcal{L})\right)^{\frac{1}{\operatorname{dim}\left(W^{\perp}\right)}},
$$

where the maximum is over lattice subspaces $W \subset \mathbb{R}^{n}$ of $\mathcal{L}$ (i.e., subspaces $W$ spanned by up to $n-1$ lattice vectors).

Proposition 5.4. Let

$$
C_{\mu}(n):=\max _{d \leq n} \sup \mu(\mathcal{L}) / \sqrt{d},
$$

where the supremum is over stable lattices $\mathcal{L} \subset \mathbb{R}^{d}$. Then, for any lattice $\mathcal{L} \subset \mathbb{R}^{n}$,

$$
\mu(\mathcal{L}) \leq \sqrt{2 e\lceil\log (2 n)\rceil} \cdot C_{\mu}(n) \cdot \mu_{\mathrm{det}}(\mathcal{L}) .
$$

Proof. Let $\{0\}=\mathcal{L}_{0} \subset \mathcal{L}_{1} \subset \cdots \subset \mathcal{L}_{k}=\mathcal{L}$ be the canonical filtration of some lattice $\mathcal{L} \subset \mathbb{R}^{n}$. Let $d_{i}:=\operatorname{rank}\left(\mathcal{L}_{i} / \mathcal{L}_{i-1}\right)$. Note that $\mathcal{L}_{i} / \mathcal{L}_{i-1}$ is a scaling of a stable lattice. In other words, $\operatorname{det}\left(\mathcal{L}_{i} / \mathcal{L}_{i-1}\right)^{-1 / d_{i}} \cdot\left(\mathcal{L}_{i} / \mathcal{L}_{i-1}\right)$ is stable. (See Item 2 of Proposition 2.5.) We therefore have by Claim 2.6 and Lemma 2.7 that

$$
\begin{aligned}
\mu(\mathcal{L})^{2} & \leq \mu\left(\bigoplus_{i} \mathcal{L}_{i} / \mathcal{L}_{i-1}\right)^{2} \\
& =\sum_{i} \mu\left(\mathcal{L}_{i} / \mathcal{L}_{i-1}\right)^{2} \\
& \leq C_{\mu}(n)^{2} \cdot \sum_{i} d_{i} \operatorname{det}\left(\mathcal{L}_{i} / \mathcal{L}_{i-1}\right)^{2 / d_{i}} .
\end{aligned}
$$

Next, we recall from Item 3 of Proposition 2.5 that the sequence $a_{i}:=\operatorname{det}\left(\mathcal{L}_{i} / \mathcal{L}_{i-1}\right)^{2 / d_{i}}$ is increasing, and we note that $\sum_{i \geq j} d_{i}=$ $\operatorname{rank}\left(\mathcal{L} / \mathcal{L}_{j-1}\right)$. We may therefore use Lemma 5.3 to bound Eq. (5) from above by

$$
\begin{aligned}
& \max _{i} \operatorname{rank}\left(\mathcal{L} / \mathcal{L}_{i}\right) \cdot \operatorname{det}\left(\mathcal{L} / \mathcal{L}_{i}\right)^{\frac{2}{\operatorname{rank}\left(\mathcal{L} / \mathcal{L}_{i}\right)}} \\
& \quad \leq \max _{W \subset \mathbb{R}^{n}} \operatorname{dim}\left(W^{\perp}\right) \cdot \operatorname{det}\left(\pi_{W^{\perp}}(\mathcal{L})\right)^{\frac{2}{\operatorname{dim}\left(W^{\perp}\right)}}
\end{aligned}
$$

as needed.

Theorem 1.5 now follows as an immediate corollary of the above results. In particular, we have $C_{\mu}(n) \leq 4(\log n+10)$ and therefore $\sqrt{2 e\lceil\log (2 n)\rceil} \cdot C_{\mu}(n) \leq 10(\log n+10)^{3 / 2}$. The result then follows from Proposition 5.4.

\subsection{Connection with the Slicing Conjecture}

In this section, we prove Theorem 5.8. The structure of the proof is based on the one suggested in [36], as was the case for the proof of our main theorem in Section 4.

As in Section 4, we are unable to work with the lattice parameter $\mu(\mathcal{L})$ that interests us directly. ${ }^{7}$ Instead, we work with the lattice parameter

$$
\bar{\mu}(\mathcal{L}):=\sqrt{\frac{1}{\operatorname{det}(\mathcal{L})} \int_{\mathcal{V}(\mathcal{L})}\|x\|^{2} \mathrm{~d} x}
$$

which, as shown in Lemma 5.5 below, gives a good approximation to $\mu$. We remark that the parameter $\bar{\mu}$ and various closely related parameters have been studied extensively (e.g., [9, 19, 21, 42]).

\footnotetext{
${ }^{7}$ While [14] give a characterization of lattices corresponding to local maxima of $\mu$, we are unable to obtain a sufficiently strong bound on the covering radius of these lattices. See [36] for more about this question.
} 
Lemma 5.5 ([21, Claim 3.1]). For any lattice $\mathcal{L} \subset \mathbb{R}^{n}$,

$$
\bar{\mu}(\mathcal{L}) \leq \mu(\mathcal{L}) \leq 2 \bar{\mu}(\mathcal{L}) .
$$

Proof. Let $t \in \mathbb{R}^{n}$ be such that $\operatorname{dist}(\boldsymbol{t}, \mathcal{L})=\mu(\mathcal{L})$. I.e., $\boldsymbol{t}$ is a "deep hole." For any $x \in \mathbb{R}^{n}$, we have

$$
\mu(\mathcal{L})=\operatorname{dist}(\boldsymbol{t}, \mathcal{L}) \leq \operatorname{dist}(\boldsymbol{x}, \mathcal{L})+\operatorname{dist}(\boldsymbol{x}+\boldsymbol{t}, \mathcal{L})
$$

(since by the triangle inequality, for all $y, z \in \mathcal{L},\|x-y\|+\| x+t-$ $z\|\geq\| t-(z-y) \|)$. Integrating, we have

$$
\begin{aligned}
\mu(\mathcal{L}) \leq & \frac{1}{\operatorname{det}(\mathcal{L})} \int_{\mathcal{V}(\mathcal{L})} \operatorname{dist}(\boldsymbol{x}, \mathcal{L}) \mathrm{d} \boldsymbol{x} \\
& \quad+\frac{1}{\operatorname{det}(\mathcal{L})} \int_{\mathcal{V}(\mathcal{L})} \operatorname{dist}(\boldsymbol{x}+\boldsymbol{t}, \mathcal{L}) \mathrm{d} \boldsymbol{x} \\
= & \frac{2}{\operatorname{det}(\mathcal{L})} \int_{\mathcal{V}(\mathcal{L})}\|\boldsymbol{x}\| \mathrm{d} \boldsymbol{x}
\end{aligned}
$$

where we have simply observed that the integral is invariant under shifts (and that, for $\boldsymbol{x} \in \mathcal{V}(\mathcal{L}), \operatorname{dist}(\boldsymbol{x}, \mathcal{L})=\|\boldsymbol{x}\|$ by definition). The result then follows by Jensen's inequality, which in particular tells us that

$$
\begin{aligned}
\left(\frac{1}{\operatorname{det}(\mathcal{L})} \cdot \int_{\mathcal{V}(\mathcal{L})}\|x\| \mathrm{d} x\right)^{2} & \leq \frac{1}{\operatorname{det}(\mathcal{L})} \cdot \int_{\mathcal{V}(\mathcal{L})}\|x\|^{2} \mathrm{~d} \boldsymbol{x} \\
& =\bar{\mu}(\mathcal{L})^{2} .
\end{aligned}
$$

We now observe that Theorem 3.1 is applicable to the function $\bar{\mu}(\mathcal{L})^{2}$. Recall that a symmetric convex body $K \subset \mathbb{R}^{n}$ is said to be isotropic if $\int_{K} \boldsymbol{x} \boldsymbol{x}^{T} \mathrm{~d} \boldsymbol{x}=\alpha \cdot I_{n}$ for some scalar $\alpha>0$.

Proposition 5.6. For any lattice $\mathcal{L} \subset \mathbb{R}^{n}$,

$$
\left.\nabla_{A} \bar{\mu}\left(A^{T} \mathcal{L}\right)^{2}\right|_{A=I_{n}}=\frac{2}{\operatorname{det}(\mathcal{L})} \int_{\mathcal{V}(\mathcal{L})} \boldsymbol{x} \boldsymbol{x}^{T} \mathrm{~d} \boldsymbol{x},
$$

where $A \in \mathrm{GL}_{n}(\mathbb{R})$ ranges over non-singular matrices. In particular, if $\mathcal{L}$ corresponds to a local maximum (or local minimum) of $\bar{\mu}(\mathcal{L})$ over the set of determinant-one lattices, then $\mathcal{V}(\mathcal{L})$ is isotropic.

Proof. To compute the gradient, we simply apply Theorem 3.1 with $f(x):=x$, and recall that

$$
\bar{\mu}\left(A^{T} \mathcal{L}\right)^{2}=\frac{1}{\operatorname{det}(\mathcal{L})} \cdot \frac{1}{|\operatorname{det}(A)|} \int_{\mathcal{V}\left(A^{T} \mathcal{L}\right)} f\left(\|x\|^{2}\right) \mathrm{d} \boldsymbol{x} .
$$

The "in particular" follows from the fact that a differentiable function $g(A)$ restricted to the set of determinant-one matrices has a critical point at $A=I_{n}$ if and only if $\left.\nabla_{A} g(A)\right|_{A=I_{n}}$ is a scalar multiple of the identity.

We define the (symmetric) isotropic constant

$$
L_{n}^{2}:=\max _{d \leq n} \frac{1}{d} \cdot \sup _{K} \int_{K}\|\boldsymbol{x}\|^{2} \mathrm{~d} \boldsymbol{x},
$$

where the supremum is taken over all isotropic symmetric convex bodies $K \subset \mathbb{R}^{d}$ of volume one. It is known to satisfy $1 /(2 \sqrt{3}) \leq$ $L_{n} \leq C n^{1 / 4}$, and the Slicing Conjecture implies that $L_{n}$ is bounded by a universal constant $[5,25]$. (The lower bound is due to the hypercube, $[-1 / 2,1 / 2]^{n}$.) We note in passing that we are only concerned with the isotropic constant for Voronoi cells, which could conceivably be easier to bound than the isotropic constant for arbitrary convex bodies.
Theorem 5.7. For any stable lattice $\mathcal{L} \subset \mathbb{R}^{n}$,

$$
\mu(\mathcal{L}) \leq 2 \bar{\mu}(\mathcal{L}) \leq 2 \sqrt{n} L_{n} .
$$

Proof. By Lemma 5.5, it suffices to prove that $\bar{\mu}(\mathcal{L}) \leq \sqrt{n} L_{n}$. Note that this is trivially true for $n=1$. We assume for induction that $\bar{\mu}\left(\mathcal{L}^{\prime}\right) \leq \sqrt{d} L_{d} \leq \sqrt{d} L_{n}$ for all stable lattices $\mathcal{L}^{\prime}$ of rank $d<n$. Recall that the set of stable lattices is compact (Item (ii) of Proposition 2.5), so that we may assume without loss of generality that $\mathcal{L}$ corresponds to a global maximum of the function $\bar{\mu}$ over this set. If this is also a local maximum over the set of determinant-one lattices, then by Proposition 5.6, the Voronoi cell is isotropic, and we have $\bar{\mu}(\mathcal{L}) \leq \sqrt{n} L_{n}$ by the definition of $\bar{\mu}$ and $L_{n}$. Otherwise, $\mathcal{L}$ must lie on the boundary of the set of stable lattices. I.e., there is some primitive sublattice $\mathcal{L}^{\prime} \subset \mathcal{L}$ of rank $0<d<n$ such that $\mathcal{L}^{\prime}$ and $\mathcal{L} / \mathcal{L}^{\prime}$ are both stable. (See Item (iv) of Proposition 2.5.) Applying the induction hypothesis and Corollary 2.8 (together with Claim 2.6), we have

$$
\begin{aligned}
\bar{\mu}(\mathcal{L})^{2} & \leq \bar{\mu}\left(\mathcal{L}^{\prime} \oplus \mathcal{L} / \mathcal{L}^{\prime}\right)^{2} \\
& =\bar{\mu}\left(\mathcal{L}^{\prime}\right)^{2}+\bar{\mu}\left(\mathcal{L} / \mathcal{L}^{\prime}\right)^{2} \leq d L_{n}^{2}+(n-d) L_{n}^{2} \\
& =n L_{n}^{2},
\end{aligned}
$$

as needed.

As far as we know, it is entirely possible that $L_{n}=1 /(2 \sqrt{3})$, i.e., that the hypercube $[-1 / 2,1 / 2]^{n}$ is the worst symmetric body for the Slicing Conjecture. If true, this would imply that for any stable $\mathcal{L} \subset \mathbb{R}^{n}, \mu(\mathcal{L}) \leq 2 \bar{\mu}(\mathcal{L}) \leq \sqrt{n / 3}$. Moreover, it is possible that the constant 2 in Lemma 5.5 can be replaced with $\sqrt{3}$, which would be tight for $\mathbb{Z}^{n}$ (this was already mentioned in [21, Conjecture 1.3]). If this is also true, then we get that for any stable $\mathcal{L} \subset \mathbb{R}^{n}$, $\mu(\mathcal{L}) \leq \sqrt{3} \bar{\mu}(\mathcal{L}) \leq \sqrt{n} / 2$, which is tight for $\mathbb{Z}^{n}$. Apart from being an interesting statement in its own right, it was shown by Shapira and Weiss [36] that such a result would imply the so-called Minkowski conjecture (see there for more information).

We can now use Proposition 5.4 to extend Theorem 5.7 to all lattices $\mathcal{L} \subset \mathbb{R}^{n}$.

TheOREM 5.8. For any lattice $\mathcal{L} \subset \mathbb{R}^{n}$,

$$
\mu(\mathcal{L}) \leq 10 \sqrt{\log n+1} \cdot L_{n} \cdot \mu_{\mathrm{det}}(\mathcal{L}) .
$$

As we observed in Footnote 4, there are lattices with $\mu(\mathcal{L}) \geq$ $C \sqrt{\log n} \cdot \mu_{\operatorname{det}}(\mathcal{L})$. So, Theorem 5.8 is tight up to a constant, assuming the Slicing Conjecture. (We made no attempt to optimize the constant in Theorem 5.8.)

\section{ACKNOWLEDGMENTS}

We are extremely grateful to Daniel Dadush for sharing his conjecture with us and for many helpful discussions. We are also indebted to Barak Weiss for introducing us to stable lattices and their salient properties and for telling us about the technique of "handling the stable local maxima separately" from his paper with Uri Shapira [36]. We also thank him for his help with the proof of Proposition 5.4. We thank Bo'az Klartag for referring us to [3] and for other useful comments. The first named author thanks Prof. Volker Kempe for his help with the two rascals. 


\section{REFERENCES}

[1] Shiri Artstein-Avidan, Apostolos Giannopoulos, and Vitali D. Milman. 2015. Asymptotic geometric analysis. Part I. Mathematical Surveys and Monographs, Vol. 202. American Mathematical Society, Providence, RI. xx+451 pages. DOI: https://doi.org/10.1090/surv/202

[2] Wojciech Banaszczyk. 1993. New bounds in some transference theorems in the geometry of numbers. Math. Ann. 296, 4 (1993), 625-635. DOI : https://doi.org/10 1007/BF01445125

[3] Sergey G. Bobkov. 2011. On Milman's ellipsoids and $M$-position of convex bodies. In Concentration, functional inequalities and isoperimetry. Contemp. Math., Vol. 545. Amer. Math. Soc., Providence, RI, 23-33. DOI : https://doi.org/10. 1090/conm/545/10762

[4] Christer Borell. 1975. The Brunn-Minkowski inequality in Gauss space. Invent. Math. 30, 2 (1975), 207-216.

[5] Jean Bourgain. 1991. On the distribution of polynomials on high-dimensional convex sets. In Geometric aspects of functional analysis (1989-90). Lecture Notes in Math., Vol. 1469. Springer, Berlin, 127-137. DOI: https://doi.org/10.1007/ BFb0089219

[6] Bill Casselman. 2004. Stability of lattices and the partition of arithmetic quotients. Asian 7. Math. 8, 4 (2004), 607-637. http://projecteuclid.org/euclid.ajm/ 1118669693

[7] Kai-Min Chung, Daniel Dadush, Feng-Hao Liu, and Chris Peikert. 2013. On the Lattice Smoothing Parameter Problem. In CCC.

[8] Henry Cohn and Abhinav Kumar. 2009. Optimality and uniqueness of the Leech lattice among lattices. Ann. of Math. (2) 170, 3 (2009), 1003-1050. DOI https://doi.org/10.4007/annals.2009.170.1003

[9] John Conway and Neil J.A. Sloane. 1998. Sphere Packings, Lattices and Groups. Springer New York. https://books.google.com/books?id=upYwZ6cQumoC

[10] Dario Cordero-Erausquin, Matthieu Fradelizi, and Bernard Maurey. 2004. The (B) conjecture for the Gaussian measure of dilates of symmetric convex sets and related problems. F. Funct. Anal. 214, 2 (2004), 410-427. DOI : https://doi.org/10. 1016/j.jfa.2003.12.001

[11] Daniel Dadush. 2012. Private communication. (2012)

[12] Daniel Dadush. 2012. Integer Programming, Lattice Algorithms, and Deterministic Volume Estimation. Ph.D. Dissertation. Georgia Institute of Technology.

[13] Daniel Dadush and Oded Regev. 2016. Towards Strong Reverse Minkowski-type Inequalities for Lattices. In FOCS. http://arxiv.org/abs/1606.06913.

[14] Mathieu Dutour Sikirić, Achill Schürmann, and Frank Vallentin. 2012. Inhomogeneous extreme forms. Ann. Inst. Fourier (Grenoble) 62, 6 (2012), 2227-2255 (2013). DOI : https://doi.org/10.5802/aif.2748

[15] T. Figiel and Nicole Tomczak-Jaegermann. 1979. Projections onto Hilbertian subspaces of Banach spaces. Israel f. Math. 33, 2 (1979), 155-171. DOI : https //doi.org/10.1007/BF02760556

[16] Daniel R. Grayson. 1984. Reduction theory using semistability. Comment. Math. Helv. 59, 4 (1984), 600-634. DOI : https://doi.org/10.1007/BF02566369

[17] Ben Green. 2007. A guest post on Terrence Tao's blog. (2007). https://terrytao.wordpress.com/2007/03/11/ ben-green-the-polynomial-freiman-ruzsa-conjecture/.

[18] P. M. Gruber and C. G. Lekkerkerker. 1987. Geometry of numbers (second ed.) North-Holland Mathematical Library, Vol. 37. North-Holland Publishing Co. Amsterdam. xvi+732 pages.

[19] Venkatesan Guruswami, Daniele Micciancio, and Oded Regev. 2005. The Complexity of the Covering Radius Problem. Comput. Complex. 14, 2 (2005), 90-121.

[20] Günter Harder and Mudumbai S. Narasimhan. 1975. On the cohomology groups of moduli spaces of vector bundles on curves. Math. Ann. 212, 3 (1975), 215-248 DOI : https://doi.org/10.1007/BF01357141

[21] Ishay Haviv, Vadim Lyubashevsky, and Oded Regev. 2009. A Note on the Distribution of the Distance from a Lattice. Discrete \& Computational Geometry 41, 1
(2009), 162-176.

[22] Martin Henk. 2002. Successive minima and lattice points. Rend. Circ. Mat. Palermo (2) Suppl. 70, part I (2002), 377-384. IV International Conference in "Stochastic Geometry, Convex Bodies, Empirical Measures \& Applications to Engineering Science", Vol. I (Tropea, 2001).

[23] G. A. Kabatjanskiı̌ and V. I. Levenšteĭn. 1978. Bounds for packings on the sphere and in space. Problemy Peredači Informacii 14, 1 (1978), 3-25.

[24] Ravi Kannan and László Lovász. 1988. Covering minima and lattice-point-free convex bodies. Ann. of Math. (2) 128, 3 (1988), 577-602. DOI : https://doi.org/10. 2307/1971436

[25] Bo'az Klartag. 2006. On convex perturbations with a bounded isotropic constant. Geom. Funct. Anal. 16, 6 (2006), 1274-1290. DOI : https://doi.org/10.1007/ s00039-006-0588-1

[26] J. C. Lagarias, H. W. Lenstra, Jr., and C.-P. Schnorr. 1990. Korkin-Zolotarev bases and successive minima of a lattice and its reciprocal lattice. Combinatorica 10, 4 (1990), 333-348. DOI : https://doi.org/10.1007/BF02128669

[27] Rafał Latała. 2002. On some inequalities for Gaussian measures. In Proceedings of the International Congress of Mathematicians, Vol. II (Beijing, 2002). Higher Ed. Press, Beijing, 813-822.

[28] D. R. Lewis. 1979. Ellipsoids defined by Banach ideal norms. Mathematika 26, 1 (1979), 18-29. DOI : https://doi.org/10.1112/S0025579300009566

[29] Shachar Lovett and Oded Regev. 2016. A counterexample to a strong variant of the Polynomial Freiman-Ruzsa conjecture in Euclidean space. (2016). http: //arxiv.org/abs/1612.03332.

[30] Daniele Micciancio and Oded Regev. 2007. Worst-case to average-case reductions based on Gaussian measures. SIAM 7. Comput. 37, 1 (2007), 267-302 (electronic). DOI : https://doi.org/10.1137/S0097539705447360

[31] Hermann Minkowski. 1910. Geometrie der Zahlen. B.G. Teubner. https://books. google.com/books?id=MusGAAAAYAAJ

[32] Hugh L. Montgomery. 1988. Minimal theta functions. Glasgow Math. 7. 30, 1 (1988), 75-85. DOI : https://doi.org/10.1017/S0017089500007047

[33] Gilles Pisier. 1982. Holomorphic semigroups and the geometry of Banach spaces. Ann. of Math. (2) 115, 2 (1982), 375-392. DOI : https://doi.org/10.2307/1971396

[34] Oded Regev and Noah Stephens-Davidowitz. 2017. A Reverse Minkowski Theorem. (2017). http://arxiv.org/abs/1611.05979.

[35] Peter Sarnak and Andreas Strömbergsson. 2006. Minima of Epstein's zeta function and heights of flat tori. Invent. Math. 165, 1 (2006), 115-151. DOI : https://doi.org/10.1007/s00222-005-0488-2

[36] Uri Shapira and Barak Weiss. 2016. Stable lattices and the diagonal group. F. Eur. Math. Soc. (JEMS) 18, 8 (2016), 1753-1767. DOI : https://doi.org/10.4171/JEMS/628

[37] Omri N. Solan. 2016. Intersections of diagonal orbits. (2016). https://arxiv.org/ abs/1612.08765.

[38] Ulrich Stuhler. 1976. Eine Bemerkung zur Reduktionstheorie quadratischer Formen. Arch. Math. (Basel) 27, 6 (1976), 604-610.

[39] V. N. Sudakov and B. S. Cirel'son. 1974. Extremal properties of half-spaces for spherically invariant measures. Zap. Naučn. Sem. Leningrad. Otdel. Mat. Inst. Steklov. (LOMI) 41 (1974), 14-24, 165. Problems in the theory of probability distributions, II.

[40] Johannes van der Corput. 1936. Verallgemeinerung einer Mordellschen Beweismethode in der Geometrie der Zahlen, Zweite Mitteilung. Acta Arithmetica 2, 1 (1936), 145-146. http://eudml.org/doc/205437

[41] Georges Voronoi. 1908. Nouvelles applications des paramètres continus à la théorie des formes quadratiques. Premier mémoire. Sur quelques propriétés des formes quadratiques positives parfaites. F. Reine Angew. Math. 133 (1908), 97-102. DOI : https://doi.org/10.1515/crll.1908.133.97

[42] Ram Zamir and Meir Feder. 1996. On lattice quantization noise. IEEE Transactions on Information Theory 42, 4 (1996), 1152-1159. 